\title{
Dynamic Beam Solutions for Real-Time Simulation and Control Development of Flexible Rockets
}

\author{
Weihua $\mathrm{Su}^{1}$ and Cecilia K. King ${ }^{2}$ \\ University of Alabama, Tuscaloosa, AL, 35487-0280 \\ Scott R. Clark ${ }^{3}$ and Edwin D. Griffin ${ }^{4}$ \\ a.i. solutions, Inc., Cape Canaveral, FL 32920 \\ and \\ Jeffrey D. Suhey ${ }^{5}$ and Michael G. Wolf ${ }^{6}$ \\ NASA Kennedy Space Center, Kennedy Space Center, FL 32899
}

\begin{abstract}
In this study, flexible rockets are structurally represented by linear beams. Both direct and indirect solutions of beam dynamic equations are sought to facilitate real-time simulation and control development for flexible rockets. The direct solution is completed by numerically integrate the beam structural dynamic equation using an explicit Newmarkbased scheme, which allows for stable and fast transient solutions to the dynamics of flexile rockets. Furthermore, in the real-time operation, the bending strain of the beam is measured by fiber optical sensors (FOS) at intermittent locations along the span, while both angular velocity and translational acceleration are measured at a single point by the inertial measurement unit (IMU). Another study in this paper is to find the analytical and numerical solutions of the beam dynamics based on the limited measurement data to facilitate the realtime control development. Numerical studies demonstrate the accuracy of these real-time solutions to the beam dynamics. Such analytical and numerical solutions, when integrated with data processing and control algorithms and mechanisms, have the potential to increase launch availability by processing flight data into the flexible launch vehicle's control system.
\end{abstract}

\section{Nomenclature}

$=$ coefficient matrix using Legendre polynomials to approximate FEM modes

$=$ beam base excitation acceleration, $\mathrm{m} / \mathrm{s}^{2}$

$=$ nodal translational acceleration in lateral direction, $\mathrm{m} / \mathrm{s}^{2}$

$=$ beam cross-section thickness, $\mathrm{m}$

$=$ beam damping matrix of FEM model

$=$ beam Young's modules, $\mathrm{Pa}$

$=$ beam bending rigidity, $\mathrm{N} \cdot \mathrm{m}^{2}$

$=$ beam load vector of FEM model

$=$ beam cross-section width, $\mathrm{m}$

$=$ beam stiffness matrix of FEM model

= beam span, $\mathrm{m}$

$=$ beam mass matrix of FEM model

$=$ beam mass per unit length, $\mathrm{kg} / \mathrm{m}$

\footnotetext{
${ }^{1}$ Assistant Professor (suw@eng.ua.edu), Dept. of Aerospace Engineering and Mechanics, Senior Member AIAA.

${ }^{2}$ Graduate Research Assistant (ckking1@crimson.ua.edu), Dept. of Aerospace Engineering and Mechanics, Student Member AIAA.

${ }^{3}$ Project Manager (scott.r.clark@nasa.gov).

${ }^{4}$ Research Scientist (edwin.d.griffin@nasa.gov).

${ }^{5}$ Flight Structures Analyst (jeffrey.d.suhey@nasa.gov).

${ }^{6}$ Research Scientist (michael.wolf@nasa.gov).
} 


$\begin{array}{ll}P_{n} & =\text { shifted Legendre polynomials } \\ p & =\text { lateral distributed load of beam, } \mathrm{N} / \mathrm{m} \\ s & =\text { IMU location, } \mathrm{m} \\ t & =\text { time, } \mathrm{s} \\ u & =\text { beam nodal displacement and rotation of FEM model } \\ w & =\text { nodal lateral displacement due to beam bending, } \mathrm{m} \\ x & =\text { spanwise position along beam, } \mathrm{m} \\ z_{0} & =\text { distance from beam reference line to locations of FOS, } \mathrm{m} \\ \alpha & =\text { damping coefficient } \\ \alpha_{1}, \alpha_{2} & =\text { tuning parameters in numerical integration } \\ \varepsilon_{x} & =\text { tensile/compressive strain due to bending } \\ \eta & =\text { magnitude of modes } \\ \theta & =\text { nodal rotation due to beam bending, rad } \\ \kappa_{y} & =\text { bending curvature, } 1 / \mathrm{m} \\ \xi & =\text { general coordinate } \\ \rho & =\text { beam material density, kg/m }{ }^{3} \\ \Phi & =\text { beam bending mode shape matrix } \\ \varphi & =\text { individual beam bending mode shape vector } \\ \omega & =\text { beam bending natural frequencies, Hz } \\ \omega_{y} & =\text { nodal angular velocity about y direction, rad } / \mathrm{s}\end{array}$

\section{Introduction}

$\mathrm{T}$

HE study of flight dynamics of rockets involves the modeling of the airframe, the propulsion, and the aerodynamic loads acting on the airframe. Traditionally, rockets are considered as rigid bodies in their flight dynamic modeling [1-5]. The 6-dof dynamic equations describing the trajectory of the rigid body are usually established by applying the Newton's Second Law or the Lagrange equation [6]. Another study [7] has modeled a rocket as an assemblage of multiple-hinged rigid bodies. Such modeling allows for considering the transvers vibration of the rocket and its aeroelastic behavior due to the interaction with the aerodynamic loads. In new developments, the sizes of new rocket designs are significantly increased, with increased propulsion power, to take more payloads. At the same time, the structural weight of the rocket airframe is also reduced, after the optimization design, to accommodate more payload and fuel. Consequently, this results in much more flexible rocket designs than ever. To take into account of the airframe flexibility, different approaches have been applied in the modeling of rocket flight dynamics, such as the linear beam approach [8] and flexible multi-body approach [9]. Moreover, adaptive control algorithms have been developed [10], where the rocket flight dynamics was also modeled using a linear beam theory.

The current work aims at developing a real-time dynamic simulation and control environment for flexible rockets so as to facilitate their effective and safe operations. The linear Euler-Bernoulli beam formulation will be used to structurally represent flexible rockets. In the development of real-time numerical beam solutions, the first need is an integration scheme for the beam dynamic equations that is stable and fast enough to allow for the realtime simulations. Implicit schemes (e.g., [11]) may provide numerically stable transient simulation results of the dynamic system. However, the sub-iterations inherently associated to these schemes prohibit them from providing the real-time simulation capability. Therefore, an explicit integration scheme will be the choice for the real-time simulation, provided that it maintains the numerical stability of the results. The explicit Newmark-based scheme developed by Chen and Ricles [12] will be selected in the current study.

On the other hand, in real-time control operations, fiber optic sensors (FOS) are used to measure the strain of a flexible body [13]. Recently, FOS system has been applied in aircraft and launch vehicle development in NASA Armstrong Flight Research Center [14] and Kennedy Space Center [15]. In control development for flexible rockets, FOS systems are able to observe the bending/torsion deformation of the airframe. It is also of interest to potentially utilize the measured structural deformation from the FOS system to control the bending/torsion vibration of the flexible rocket, with appropriately designed control algorithms. Moreover, if the solution of the beam dynamics is properly developed, a rapid processing of the FOS measurement data may enable a real-time control mechanism for flexible rockets.

However, at a specific real-time point, the excitation to the rocket may not be available from the measurement, even though the intermittent bending strain along the rocket can be measured by FOS system. Additionally, both the 
angular velocity and translational acceleration can be measured at a single point by the inertial measurement unit (IMU) to provide some dynamic characteristics of the rocket. Such information will be the only input of an indirect solution of the beam dynamics, from which the angular velocity and translational acceleration along the beam will need to be obtained. Figure 1 illustrates the distribution of the sensors and measurements along a beam representation of a flexible rocket. This solution process will be quite different from the direct integration of the beam dynamic equations using the explicit scheme. Moreover, an analytical solution to the angular velocity and translational acceleration are required to facilitate further real-time control development.

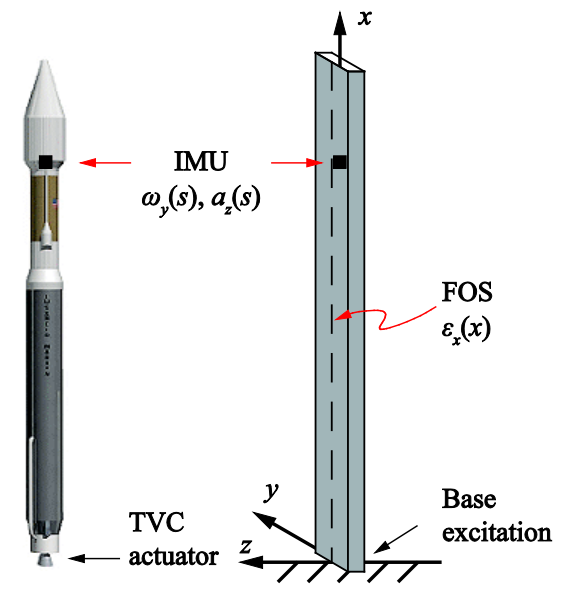

Figure 1 Beam representation of flexible rocket and the distribution of sensor measurements.

Figure 2 illustrates the two dynamic solutions to be developed in the paper, where the two yellow blocks need to be realized in a real-time sense, while the control development is not included in the current study. The analytical solution and the numerical implementation of this work will have the potential to increase launch availability by processing real-time flight data (including the deformation and kinematics) into the flexible launch vehicle's control system. As a research supported by the NASA Launch Services Program, this work is beneficial to the future of NASA and the aerospace industry.

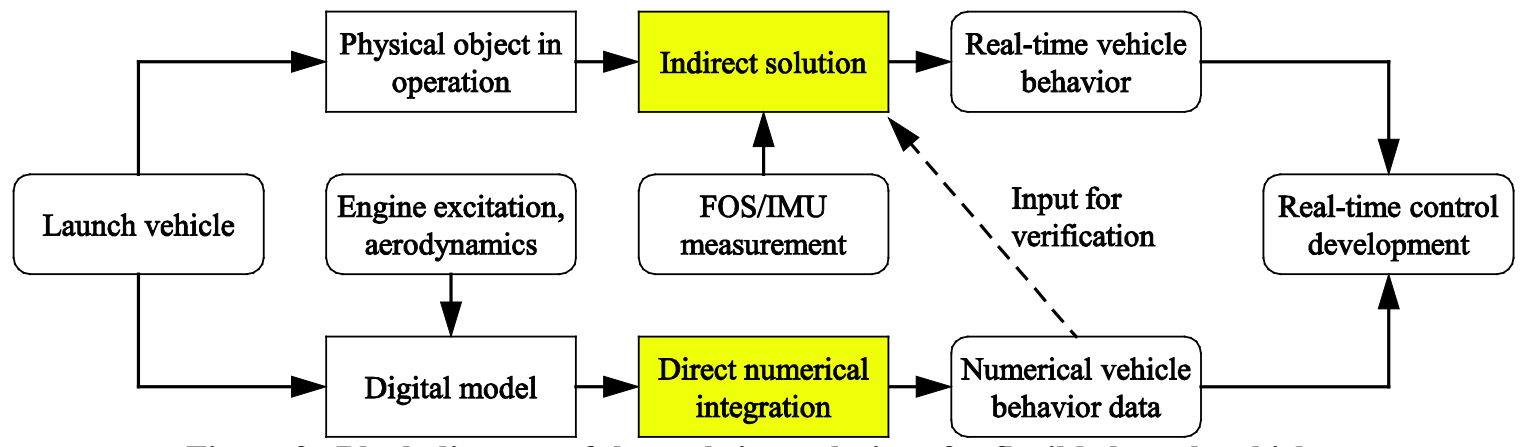

Figure 2 Block diagram of the real-time solutions for flexible launch vehicles.

\section{Theoretical Formulation}

Flexible rockets are modeled as linear beams, by taking advantage of their geometry. A specific constraint to the current study is that the beam dynamic responses should be solved in a real-time scale. Therefore, the solutions of the beam formation need some special treatment, which are described as follows.

\section{A. Euler-Bernoulli Beam Equation of Motion}

In the current study, a flexible rocket is modeled using the classic Euler-Bernoulli beam formulation, subject to a base acceleration excitation $a_{B}(t)$. As shown in Fig. 3, the flexible rocket is treated as a cantilever beam in a moving frame $(x y z)$ that is fixed at the root. The lateral distributed force $p(x, t)$ is derived from the base acceleration: 


$$
p(x, t)=m(x) a_{B}(t)
$$

where $m(x)$ is the mass per unit length of the beam. Only the flatwise bending about the $y$ axis is considered. The equation of motion for the beam is given as

$$
\left(E I_{y}(x) w^{\prime \prime}(x, t)\right)^{\prime \prime}+m(x) \ddot{w}(x, t)=p(x, t)
$$

where $w(x, t)$ is the beam's lateral displacement relative to the moving frame $x y z$ and $E I_{y}(x)$ is its bending rigidity about the $y$ axis. Note that (") denotes the second time derivative, while ()' and ()" denote spatial partial derivatives of the corresponding variable. In general, the inertial and rigidity properties are not uniform along the beam span. The cantilever boundary condition requires the following relations to be satisfied:

$$
\begin{array}{rlrl}
w(0) & =0 & E I_{z} w^{\prime \prime}(L) & =0 \\
w^{\prime}(0) & =0 & \left(E I_{z} w^{\prime \prime}(L)\right)^{\prime} & =0
\end{array}
$$

where $L$ is the beam span. The finite element approach is used to solve the governing equation. The finite element model of Euler-Bernoulli beams is well established and can be found in various literatures, which is given as

$$
[M]\{\ddot{u}\}+[C]\{\dot{u}\}+[K]\{u\}=\{F\}
$$

where the stiffness-proportional damping matrix is determined by

$$
[C]=\alpha[K]
$$

Each node has a displacement $(w)$ and a rotation $(\theta)$ degree of freedom, i.e.,

$$
\left\{u_{i}\right\}=\left\{\begin{array}{l}
w_{i} \\
\theta_{i}
\end{array}\right\}
$$

The inertia $[M]$ and stiffness $[K]$ matrices are obtained from the assemblage of the elemental matrices. Note that the finite element model is compatible to both uniform and non-uniform beams. Concentrated inertias, if exist, can be attached to the corresponding nodes in the finite element model.

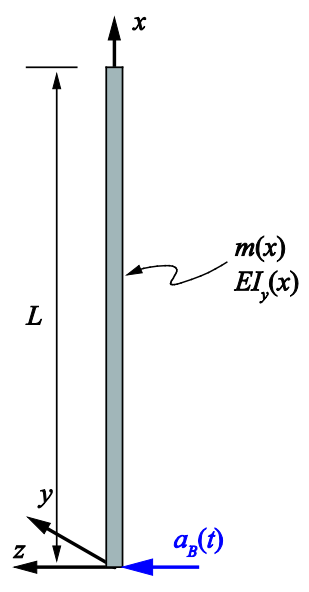

(a)

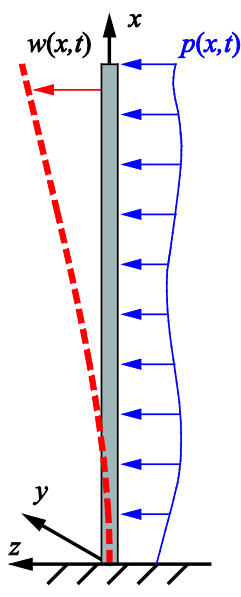

(b)

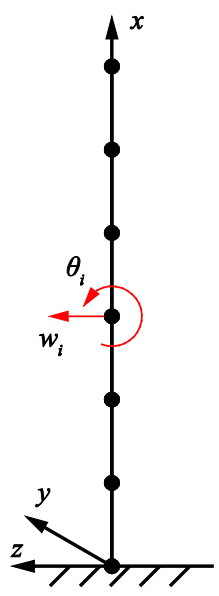

(c)

Figure 3 Description of a continous beam and its finite element discretization. 


\section{B. Kinematics}

According to the kinematics, the tensile strain due to the beam bending is related to the nodal displacement as

$$
\varepsilon_{x}(x, t)=-z_{0} w^{\prime \prime}(x, t)
$$

where $z_{0}$ is the distance from the beam reference line (centerline for this study) to locations of the FOS, where the strains are measured, which are usually the surface of the beam. Additionally, the angular velocity and translational acceleration are obtained as

$$
\begin{aligned}
a_{z}(x, t) & =\ddot{w}(x, t) \\
\omega_{y}(x, t) & =\dot{\theta}_{y}(x, t)=\dot{w}^{\prime}(x, t)
\end{aligned}
$$

\section{Normal Modes and Approximation using Continuous Functions}

In a modal analysis, the beam deformation is considered as a linear combination of the linear normal modes, given as

$$
w(x, t)=\sum_{j=1}^{\infty} \varphi_{j}(x) \eta_{j}(t)=\left[\Phi_{w}\right]\{\eta\}
$$

where $\varphi_{i}$ are the linear normal modes of the beam, obtained from the eigenvalue solution of Eq. (4) and $\eta_{i}$ are the magnitudes of the corresponding modes varying in time. For an approximate solution, one may truncate the series and select only the first $n$ modes.

However, the normal modes derived from the eigenvalue solution of Eq. (4) are discrete, which are represented by the eigen-displacement and rotation at each node of the finite element model. There are no analytical functions of the mode shapes that are directly available from the eigenvalue solution of Eq. (4). To allow the spatial derivatives of the mode shapes in Eqs. (7) and (8), the discrete mode shapes can be approximated by using some analytical functions. In the current study, this is done by using the shifted Legendre polynomials [16], which are a set of complete and orthogonal polynomials defined in the domain of $[0,1]$. The general equations for the shifted Legendre polynomials are given as

$$
P_{0}(x)=1, \quad P_{1}(x)=2 x-1, \quad P_{i+1}(x)=\frac{(2 i+1)(2 x-1) P_{i}(x)-i P_{i-1}(x)}{i+1}
$$

The first few shifted Legendre polynomials are plotted in Fig. 4.

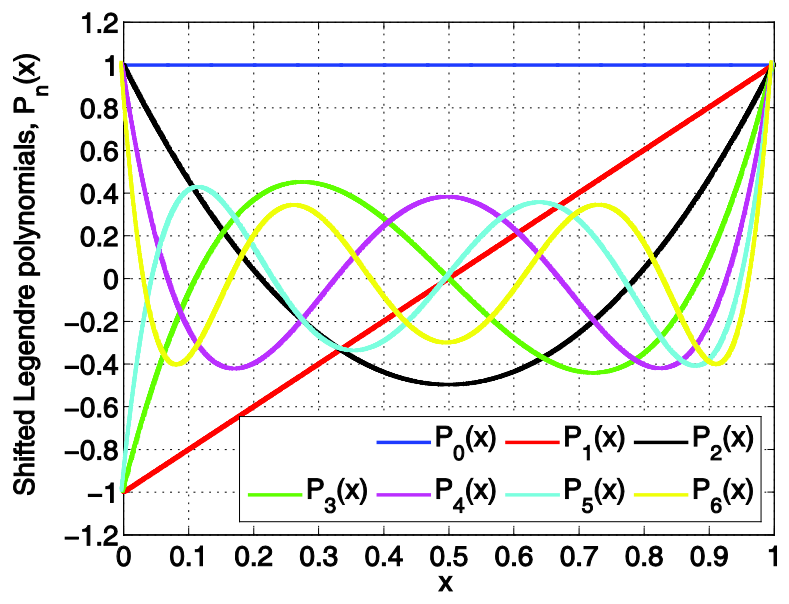

Figure 4 First seven shifted Legendre polynomials. 
Linear combinations of Legendre polynomials can be used to fit any functions. For a discrete mode shape $\varphi_{j}$ that is solved from Eq. (4), it can be fitted by the linear combinations of the first $m+1$ shifted Legendre polynomials:

$$
\varphi_{j w}=\sum_{i=0}^{\infty} a_{i j} P_{i}(x) \approx a_{0 j} P_{0}(x)+a_{1 j} P_{1}(x)+\cdots+a_{m j} P_{m}(x)
$$

where $\varphi_{j w}$ are the displacement components of the $j$ th mode and $P_{m}(x)$ are all evaluated at the nodal coordinates of the finite element model. The coefficients $a_{m j}$ are to be determined. This approximation can be done for all modes retained in Eq. (9) after the truncation, using the same set of the shifted Legendre polynomials. Obviously, the corresponding coefficients $a_{m j}$ are different for different modes $\varphi_{j w}$. This approximation is represented as

$$
\begin{aligned}
{\left[\Phi_{w}(x)\right] } & =\left[\begin{array}{llll}
\varphi_{1 w} & \varphi_{2 w} & \cdots & \varphi_{n w}
\end{array}\right] \\
& =\left[\begin{array}{lllll}
P_{0}(x) & P_{1}(x) & P_{2}(x) & \cdots & P_{m}(x)
\end{array}\right]\left[\begin{array}{cccc}
a_{01} & a_{02} & \cdots & a_{0 n} \\
a_{11} & a_{12} & \cdots & a_{1 n} \\
a_{21} & a_{22} & \cdots & a_{2 n} \\
\vdots & \vdots & \cdots & \vdots \\
a_{m 1} & a_{m 2} & \cdots & a_{m n}
\end{array}\right] \\
& =[P(x)][A]
\end{aligned}
$$

Therefore, the coefficient matrix $[A]$ is solved by

$$
[A]=[P(x)]^{-1}\left[\Phi_{w}(x)\right]
$$

with given mode shapes $\left[\Phi_{w}\right]$. Note that the Moore-Penrose pseudo-inverse of the $[P(x)]$ matrix will be used, as it is generally invertible. Once $[A]$ is determined, mode shapes of nodal rotations are obtained by taking the derivative of $[P]$, such that

$$
\left[\Phi_{\theta}(x)\right]=\left[\begin{array}{llll}
\varphi_{1 \theta} & \varphi_{2 \theta} & \cdots & \varphi_{n \theta}
\end{array}\right]=\left[P^{\prime}(x)\right][A]
$$

Consequently, the spatial derivatives of the beam nodal displacement are obtained by differentiating the polynomials $P(x)$, given as

$$
\begin{aligned}
w(x, t) & =[P(x)][A]\{\eta(t)\} \\
w^{\prime}(x, t) & =\theta_{y}(x, t)=\left[P^{\prime}(x)\right][A]\{\eta(t)\} \\
w^{\prime \prime}(x, t) & =-\kappa_{y}(x, t)=\left[P^{\prime \prime}(x)\right][A]\{\eta(t)\}
\end{aligned}
$$

From Eqs. (7), (8), and (15), one can find the strain, angular velocity, and translational acceleration as follows:

$$
\begin{aligned}
\varepsilon_{x}(x, t) & =-z_{0}\left[P^{\prime \prime}(x)\right][A]\{\eta(t)\} \\
\omega_{y}(x, t) & =\left[P^{\prime}(x)\right][A]\{\dot{\eta}(t)\} \\
a_{z}(x, t) & =[P(x)][A]\{\ddot{\eta}(t)\}
\end{aligned}
$$

\section{Modal Transformation of Equation of Motion}

To reduce the number of degrees of freedom and save the time in real-time transient simulations, a modal transformation is performed with Eq. (4). To be consistent with the transformation of mode shapes into the combinations of the shifted Legendre polynomials, complete mode shapes from the finite element equation (consisting of both nodal displacements and rotations) are represented as 


$$
[\Phi(x)]=[\bar{P}(x)][A]
$$

where each column of $[\bar{P}(x)]$ is formed by alternate components from $P(x)$ and $P^{\prime}(x)$. Therefore, the finite element solution is represented by the shifted Legendre polynomials as

$$
\{u\}=[\Phi(x)]\{\eta\}=[\bar{P}(x)][A]\{\eta\}
$$

Substitute Eq. (18) into Eq. (4) and pre-multiply $[A]^{T}[\bar{P}]^{T}$ on both sides of the equation, it yields the modal-based equation of motion:

$$
[\bar{M}]\{\ddot{\eta}\}+[\bar{C}]\{\dot{\eta}\}+[\bar{K}]\{\eta\}=\{\bar{F}\}
$$

where

$$
\begin{array}{cc}
{[\bar{M}]=[A]^{T}[\bar{P}]^{T}[M][\bar{P}][A]} & {[\bar{C}]=[A]^{T}[\bar{P}]^{T}[C][\bar{P}][A]} \\
{[\bar{K}]=[A]^{T}[\bar{P}]^{T}[K][\bar{P}][A]} & \{\bar{F}\}=[A]^{T}[\bar{P}]^{T}\{F\}
\end{array}
$$

The time-domain transient analysis of the flexible rocket can be done with either Eq. (4) or Eq. (19). However, Eq. (19) usually involves significantly less degrees of freedom than Eq. (4).

\section{E. Direct Solution}

The numerical integration of the equation of motion (Eq. (4) or Eq. (19)) is needed in the current study to obtain the transient response of the flexible rocket. An explicit numerical integration scheme is selected over implicit approaches to provide fast solutions of the beam dynamic response, facilitating the real-time simulations. The explicit integration scheme developed by Chen and Ricles $[12,17]$ is implemented here, which has been proved to be unconditionally stable [17]. This will allow for the use of relatively larger time steps in transient solutions, while keeping the numerical stability. For a second order equation of motion (Eq. (4) or (19)), the "velocity" and "displacement" at each time step are determined by

$$
\begin{aligned}
& \dot{\xi}_{i+1}=\dot{\xi}_{i}+\alpha_{1} \ddot{\xi}_{i} \Delta t \\
& \xi_{i+1}=\xi_{i}+\dot{\xi}_{i} \Delta t+\alpha_{2} \ddot{\xi}_{i} \Delta t^{2}
\end{aligned}
$$

where

$$
\alpha_{1}=\alpha_{2}=\frac{4[\tilde{M}]}{4[\tilde{M}]+2[\tilde{C}] \Delta t+[\tilde{K}] \Delta t^{2}}
$$

In the equations, $\xi$ is the general coordinate of either $u$ or $\eta$ and the matrices with "tilde" are general mass, damping and stiffness matrices from either Eq. (4) or Eq. (19).

\section{F. Indirect Solution}

Another problem that is to be tackled in the current study is the situation in a real-time operation when the base excitation $a_{B}$ is unknown, while limited sensor measurements of the strain and kinematic quantities are available. This capability to find the beam dynamic solution under such a condition is particularly important in the real-time control of launch vehicles. In this case, the direct integration of the equation of motion (Eq. (4) or Eq. (19)) is not feasible. However, an indirect solution of the modal magnitude $\eta(t)$ can be obtained based on the available strain measurements $\varepsilon_{x}(x, t)$ from the FOS along the beam, subject to the constraints of the inertial measurements $\left(\omega_{z}(s, t)\right.$ and $\left.a_{y}(s, t)\right)$ from the IMU at a single location of $x=s$. 


\section{Strain from FOS}

If $\varepsilon_{x}(x, t)$ is measured by the FOS, the instantaneous modal magnitudes $\eta(t)$ are solved from the first equation of Eq. (16):

$$
\left[B_{1}\right]\{\eta\}=\left\{D_{1}\right\}
$$

where

$$
\begin{aligned}
& {\left[B_{1}\right]=-z_{0}\left[P^{\prime \prime}\right][A]} \\
& \left\{D_{1}\right\}=\varepsilon_{x}(x, t)
\end{aligned}
$$

It can be seen that $B_{1}$ carries the system's modal information and $D_{1}$ consists of the instantaneous measurement by the FOS.

2. Angular Velocity from IMU

If $\omega_{y}(s, t)$ is measured by the IMU at $x=s$, the instantaneous modal magnitudes $\eta(t)$ should satisfy the following relation derived from Eq. (16):

$$
\left\{\bar{B}_{2}\right\}\{\dot{\eta}(t)\}=\bar{D}_{2}
$$

where

$$
\begin{aligned}
\left\{\bar{B}_{2}\right\} & =\left\{P^{\prime}(s)\right\}[A] \\
\bar{D}_{2} & =\omega_{y}(s, t)
\end{aligned}
$$

A backward finite difference is used to find the rate of the modal magnitude, i.e.,

$$
\dot{\eta}(t)=\dot{\eta}_{t}=\frac{\eta_{t}-\eta_{t-\Delta t}}{\Delta t}
$$

Substitute Eq. (27) in Eq. (25), it yields

$$
\left\{B_{2}\right\}\{\eta(t)\}=D_{2}
$$

where

$$
\begin{aligned}
\left\{B_{2}\right\} & =\left\{\bar{B}_{2}\right\} \\
D_{2} & =\bar{D}_{2} \Delta t+\left\{\bar{B}_{2}\right\}\left\{\eta_{t-\Delta t}\right\}
\end{aligned}
$$

Obviously, it requires knowing the history of the solution in order to solve the magnitude $\eta(t)$. In practice, the size of $\Delta t$ can be decided by the sampling frequency of the FOS.

3. Translational Acceleration from IMU

If $a_{z}(s, t)$ is measured by the IMU at $x=s$, the instantaneous modal magnitudes $\eta(t)$ should also satisfy the following relation derived from Eq. (16):

$$
\left\{\bar{B}_{3}\right\}\{\ddot{\eta}(t)\}=\bar{D}_{3}
$$

where 


$$
\begin{aligned}
\left\{\bar{B}_{3}\right\} & =\{P(s)\}[A] \\
\bar{D}_{3} & =a_{z}(s, t)
\end{aligned}
$$

The approximation of the acceleration of $\eta$ using the backward finite-difference scheme is

$$
\ddot{\eta}_{t}=\frac{\eta_{t}-2 \eta_{t-\Delta t}+\eta_{t-2 \Delta t}}{\Delta t^{2}}
$$

Eqs. (30) and (32) result in

$$
\left\{B_{3}\right\}\{\eta(t)\}=D_{3}
$$

where

$$
\begin{aligned}
\left\{B_{3}\right\} & =\left\{\bar{B}_{3}\right\} \\
D_{3} & =\bar{D}_{3} \Delta t^{2}+\left\{\bar{B}_{3}\right\}\left\{2 \eta_{t-\Delta t}-\eta_{t-2 \Delta t}\right\}
\end{aligned}
$$

\section{Combined Solution}

One can solve for the current modal magnitude $\eta(t)$ that satisfy the measurements of both FOS and IMU by combining Eqs. (24), (28), and (33), which is

$$
\{\eta\}=[B]^{-1}\{D\}
$$

where

$$
\begin{aligned}
& {[B]^{T}=\left[\begin{array}{lll}
B_{1}^{T} & B_{2}^{T} & B_{3}^{T}
\end{array}\right]} \\
& \{D\}^{T}=\left\{\begin{array}{lll}
D_{1}^{T} & D_{2}^{T} & D_{3}^{T}
\end{array}\right\}
\end{aligned}
$$

In practice, a pseudo inverse of $B$ matrix is required, since it is generally not invertible.

5. Estimation of Spanwise Angular Velocity and Translational Acceleration

In the last step, one needs to estimate the angular velocity and translational acceleration along the beam reference line based on the solution of $\eta$ and the kinematics. From Eq. (16), the spanwise angular velocity and translational acceleration are obtained as

$$
\begin{aligned}
& \omega_{y}(x, t)=\left[P^{\prime}(x)\right][A]\{\dot{\eta}(t)\}=\frac{1}{\Delta t}\left[P^{\prime}(x)\right][A]\left\{\eta_{t}-\eta_{t-\Delta t}\right\} \\
& a_{z}(x, t)=[P(x)][A]\{\ddot{\eta}(t)\}=\frac{1}{\Delta t^{2}}[P(x)][A]\left\{\eta_{t}-2 \eta_{t-\Delta t}+\eta_{t-2 \Delta t}\right\}
\end{aligned}
$$

\section{Numerical Studies}

Both the direct and indirect real-time solutions of a representative beam model are presented in this section. Accuracy of the solutions will be discussed based on the simulation data.

\section{A. Approximate Mode Shapes of a Flexible Beam}

Geometrical and material properties of a beam model are listed in Table 1. The cross-section of the beam is rectangular. The fiber optical sensors are assumed to be attached on the wider surface. Therefore, the distance of the sensors to the beam reference line in the current study is $z_{0}=b / 2$. 
The natural frequencies and discrete mode shapes of the beam are firstly calculated using the finite element model, with the mesh being refined. Table 2 and Fig. 5 compare the natural frequencies obtained from these finite element models and the analytical solutions obtained by solving the characteristic equation of the continuous uniform beam. If one needs the relative error of the first five modes (below $100 \mathrm{~Hz}$, see Table 2) to be less than $0.1 \%$, a 14-element mesh of the beam is sufficient. However, the purpose of the finite element model and the eigenvalue solution is to provide the discrete mode shapes to be fitted by the Legendre polynomials. A finer mesh may improve the quality of the fitted modes. For this reason, a 20-element mesh of the beam is used. Once the finite element model is created, the corresponding number of Legendre polynomials should be properly selected. Figure 4 plots the fitted modes of the beam using 26 Legendre polynomials based on the 20-element mesh. Even though most of data points are well fitted, the root and tip regions see large discrepancy between the fitted modes and those from the FEM solution. This is because that the higher-order Legendre polynomials have larger slopes as the two ends. Thus, they need more data points for a proper fit. Figure 7 compares the good fit with 20 Legendre polynomials. The analytical solutions of the mode shapes are also plotted to verify the accuracy of the fitted modes.

One more verification is to check the derivatives of the fitted modes. For the cantilever beam in the current study, the free end has no applied force or moment. Therefore, the beam curvature and its slope at the free end must be zeros, which has been captured by the fitted modes (in terms of bending curvatures), as seen in Fig. 8 .

It is of interest to note that the shifted Legendre polynomials can also be used to approximate mode shapes of other beam configurations. Figure 9 demonstrates that the mode shapes of a uniform beam with a free-free boundary condition can be correctly fitted. Figure 10 illustrates how accurately the modes of a non-uniform beam are approximated, where the stiffness of the outer half board of the beam is reduced to the half of the nominal one.

Table 1. Properties of a uniform beam

\begin{tabular}{lll}
\hline \hline Property & Value & Unit \\
\hline Span, $L$ & 1.575 & $\mathrm{~m}$ \\
Cross-section thickness, $b$ & $4.826 \times 10^{-3}$ & $\mathrm{~m}$ \\
Cross-section width, $h$ & $2.543 \times 10^{-2}$ & $\mathrm{~m}$ \\
Material density, $\rho$ & $2.666 \times 10^{3}$ & $\mathrm{~kg} / \mathrm{m}^{3}$ \\
Young's Modules, $E$ & $6.350 \times 10^{10}$ & $\mathrm{~Pa}$ \\
\hline \hline
\end{tabular}

Table 2. Natural frequencies $(\mathrm{Hz})$ of the uniform cantilever beam

\begin{tabular}{c|cccc|c}
\hline \hline & \multicolumn{3}{|c|}{ Finite Element Solution } & Analytical \\
\cline { 2 - 4 } & 5 Elements & 10 Elements & 14 Elements & 20 Elements & Solution \\
\hline 1 & 1.5343 & 1.5343 & 1.5343 & 1.5343 & 1.5343 \\
2 & 9.6201 & 9.6156 & 9.6154 & 9.6153 & 9.6153 \\
3 & 27.0198 & 26.9300 & 26.9249 & 26.9236 & 26.9231 \\
4 & 53.3773 & 52.8089 & 52.7721 & 52.7619 & 52.7586 \\
5 & 88.5930 & 87.4336 & 87.2738 & 87.2285 & 87.2137 \\
6 & 147.1774 & 130.9854 & 130.4786 & 130.3310 & 130.2822 \\
7 & 215.2480 & 183.7789 & 182.4877 & 182.0960 & 181.9644 \\
8 & 312.1570 & 246.2130 & 243.4629 & 242.5671 & 242.2602 \\
\hline \hline
\end{tabular}




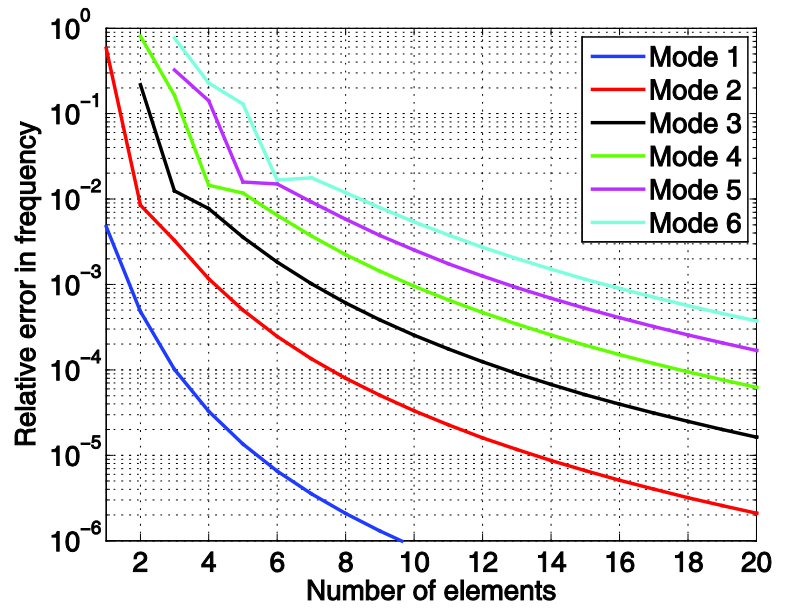

Figure 5 Relative errors of natural frequencies from the finite element solutions, compared with the analytical solutions.
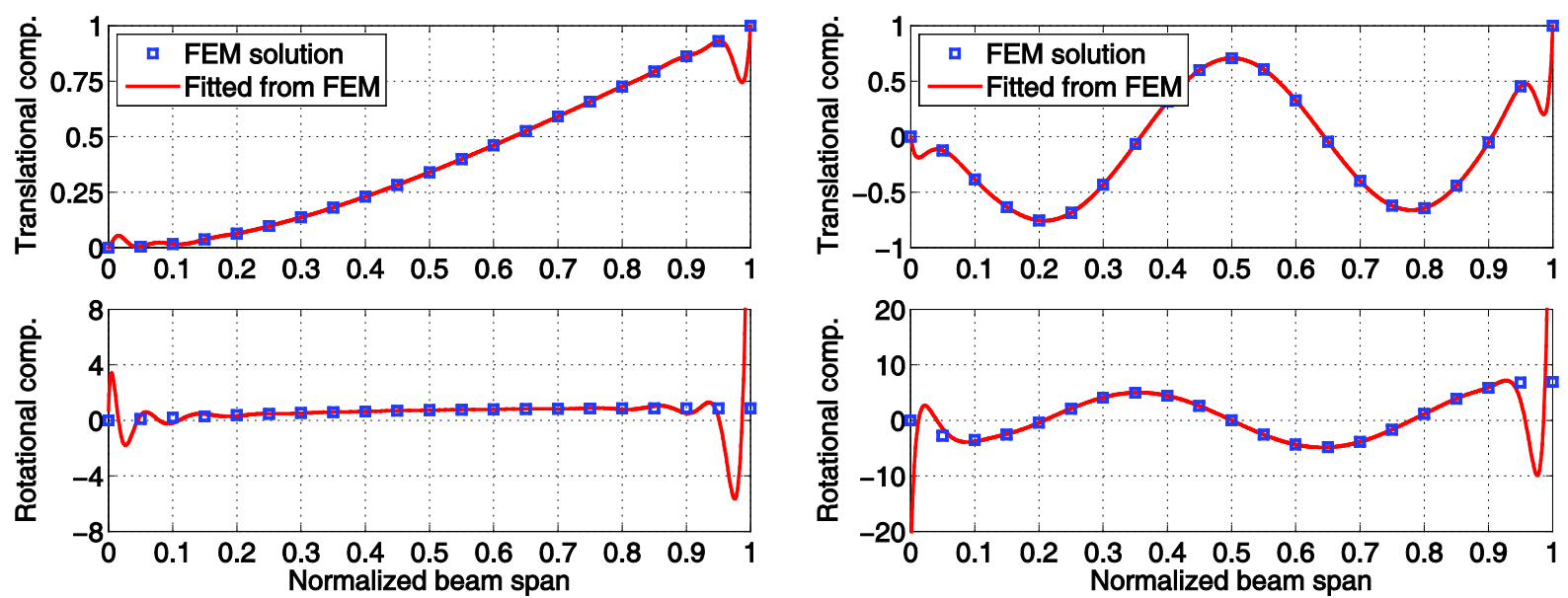

(a) First bending mode

(b) Fourth bending mode

Figure 6 Improperly fitted mode shapes using excessive Legendre polynomials.
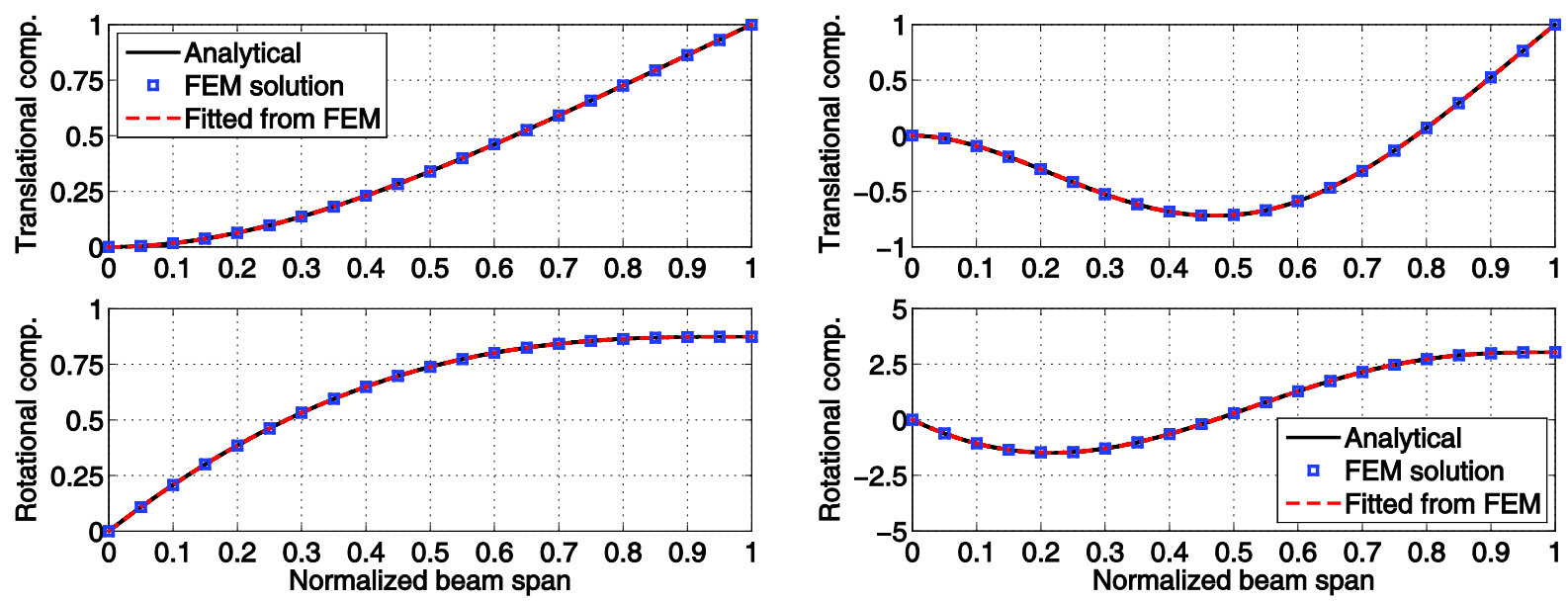

(a) First bending mode

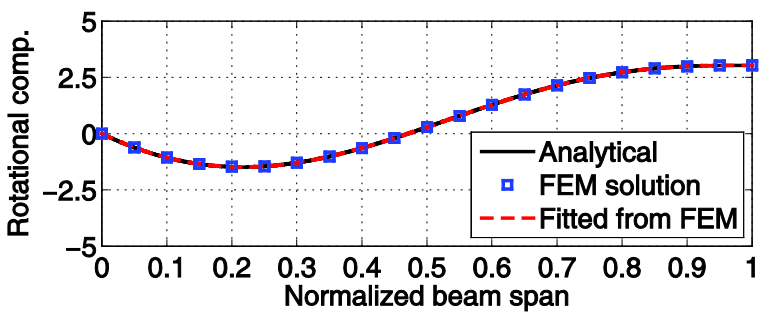

(b) Second bending mode

11

American Institute of Aeronautics and Astronautics 

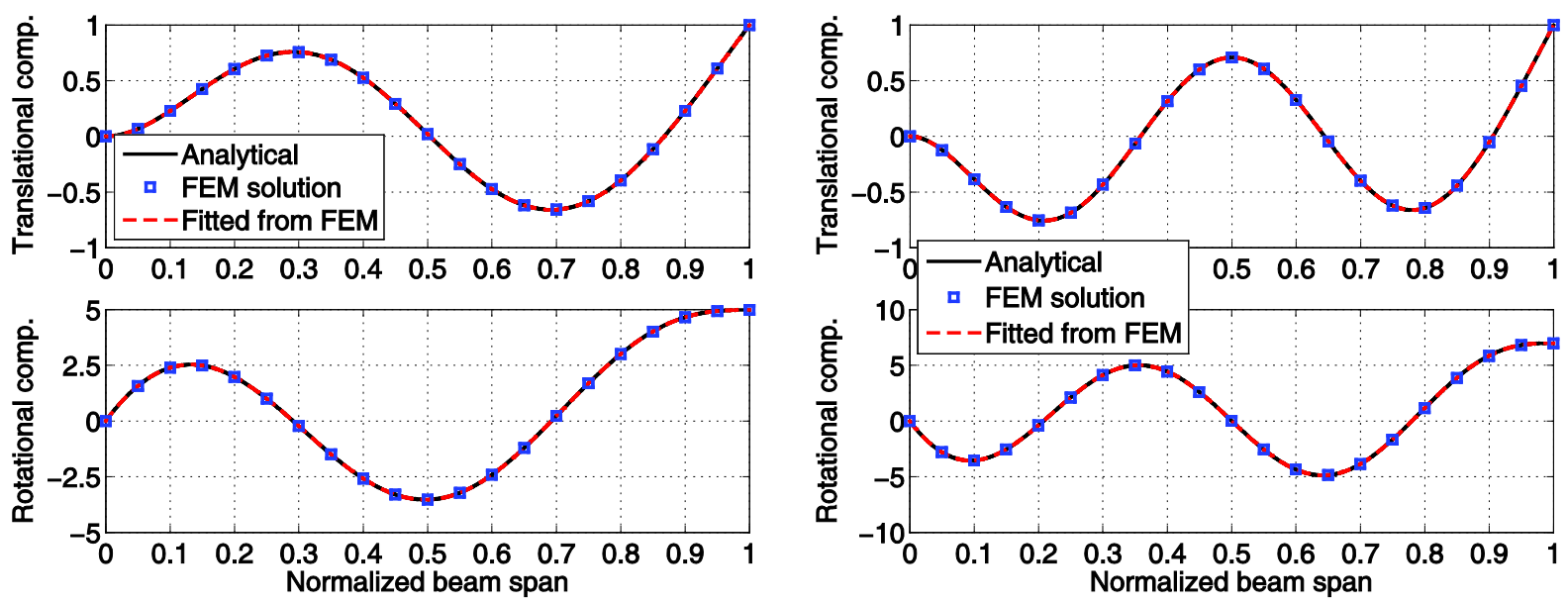

(c) Third bending mode

(d) Fourth bending mode

Figure 7 Cantilever beam bending mode shapes from analytical and finite element (20 elements) solutions and the fitted continuous shape (using 20 shifted Legendre polynomials).

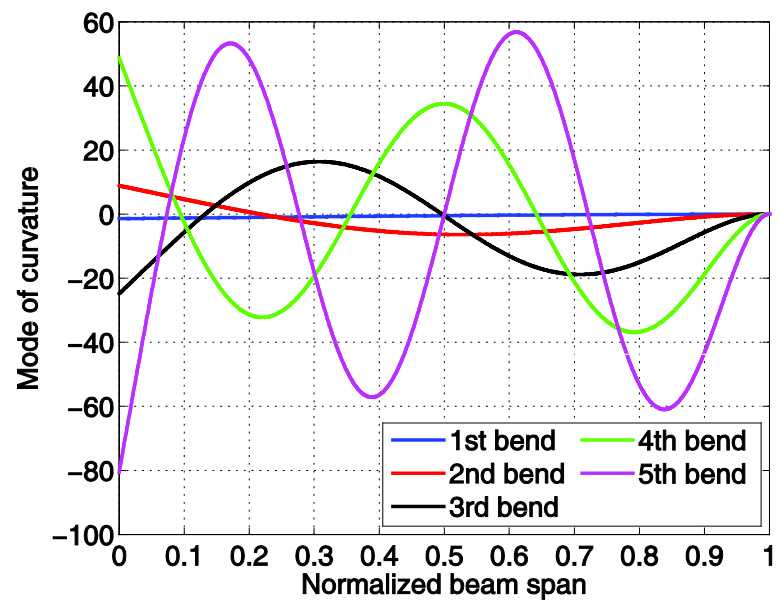

Figure 8 First five curvature modes.
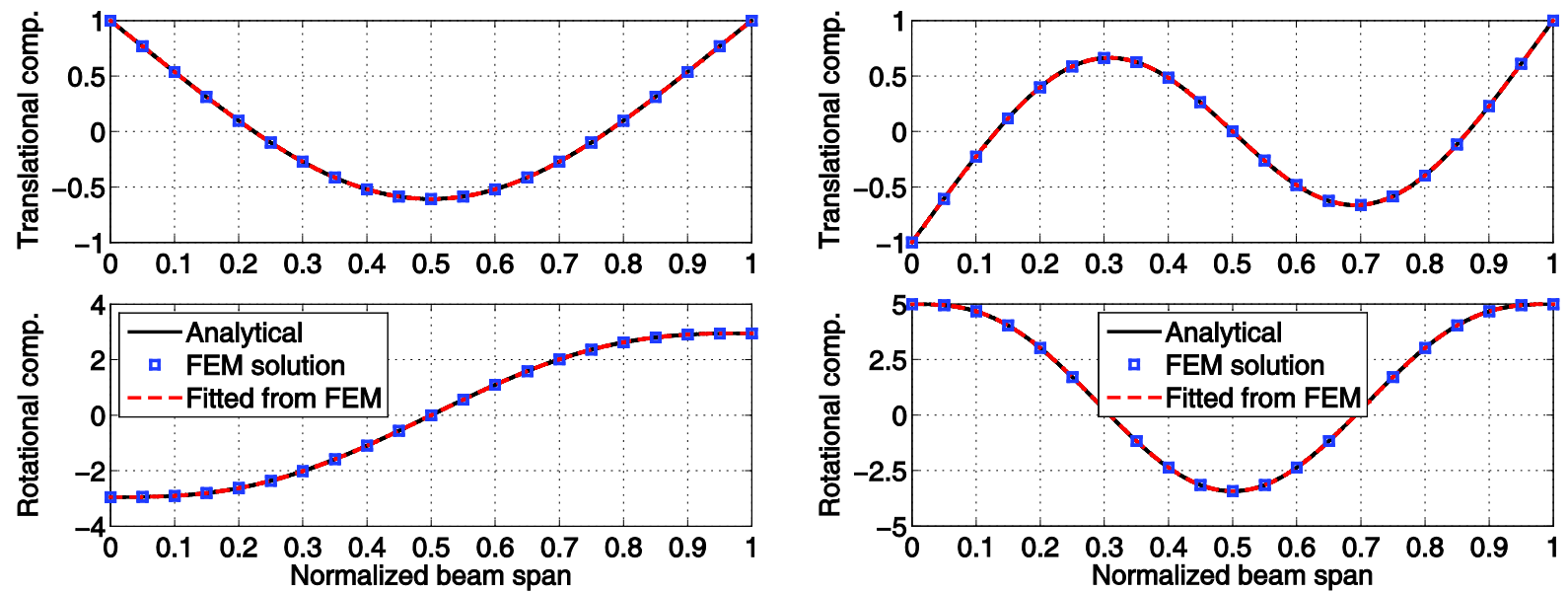

(a) First symmetric bending mode

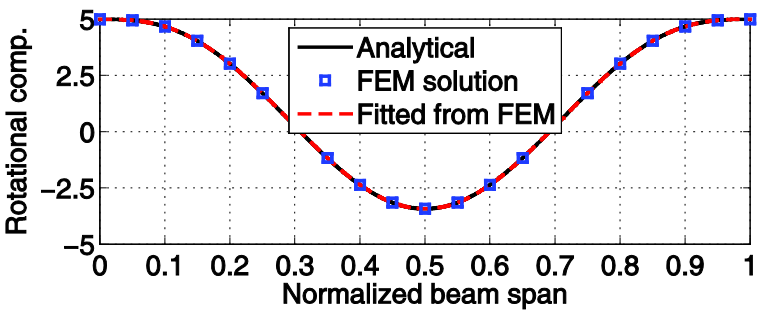

(b) First anti-symmetric bending mode

Figure 9 Free-free bending mode shapes from analytical and finite element (20 elements) solutions and the fitted continuous shape (using 20 shifted Legendre polynomials).

12

American Institute of Aeronautics and Astronautics 

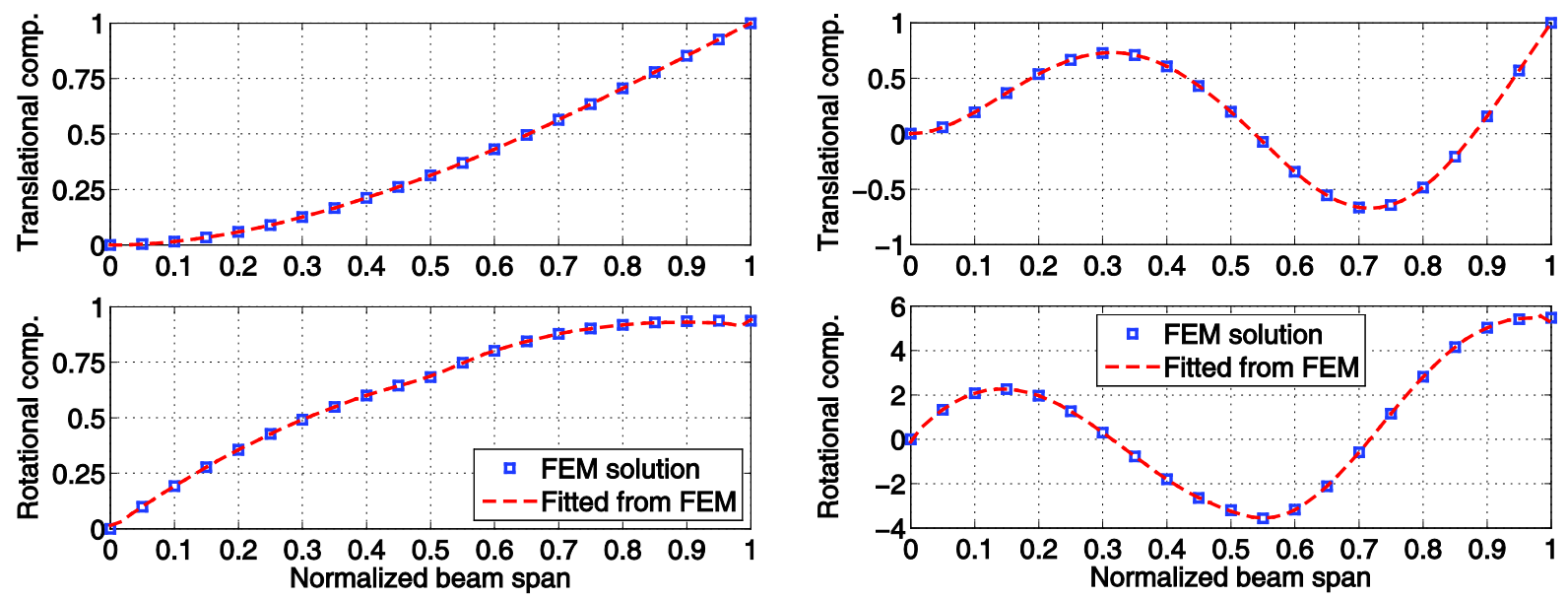

(a) First bending mode

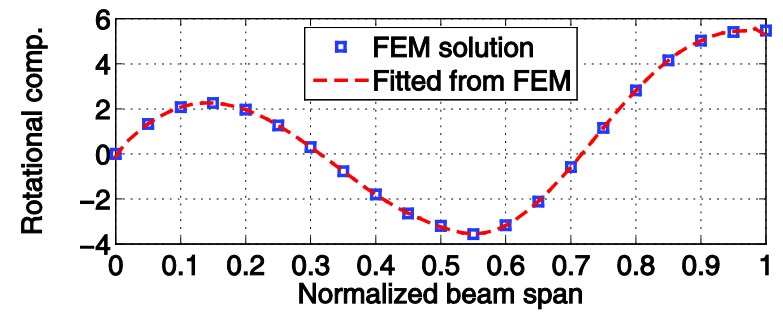

(b) Third bending mode

Figure 10 Cantilever non-uniform beam bending mode shapes from finite element (20 elements) solution and the fitted shape (using 20 shifted Legendre polynomials).

\section{B. Direct Time Integration}

In this section, the root acceleration excitation of the beam is assumed to be a sinusoidal function of $a_{B}=$ $10 \sin (10 \pi t) \mathrm{m} / \mathrm{s}^{2}$. The Chen-Ricles scheme from Refs. $[12,17]$ is implemented for the numerical integrations of the beam equations. A first study is to numerically integrate the finite-element based equation Eq. (4), where the beam is divided into 20, 50, 100, and 200 elements, respectively. Figure 11 compares the resulting beam tip displacement and translational acceleration in the lateral direction using the models of different meshes. In all simulations, the time step is $0.01 \mathrm{~s}$, which is selected based on the sampling frequency of FOS. Table 3 compares the CPU time cost of each solution. Overall, all the solutions provide very close results of displacement. They may show some discrepancy in the acceleration results from Fig. 11. As the translational acceleration will be an important quantity to be used for the real-time control development, it is desired to use a finer mesh to ensure a more accurate solution of the acceleration. However, the finite element solution using 200 elements, which is believed to be the closest to the true solution, is far from satisfying the requirement of the "real-time" solutions. In fact, only the 20 -element model can be used for real-time studies, as observed from Table 3 . However, its solution accuracy may not be satisfactory.
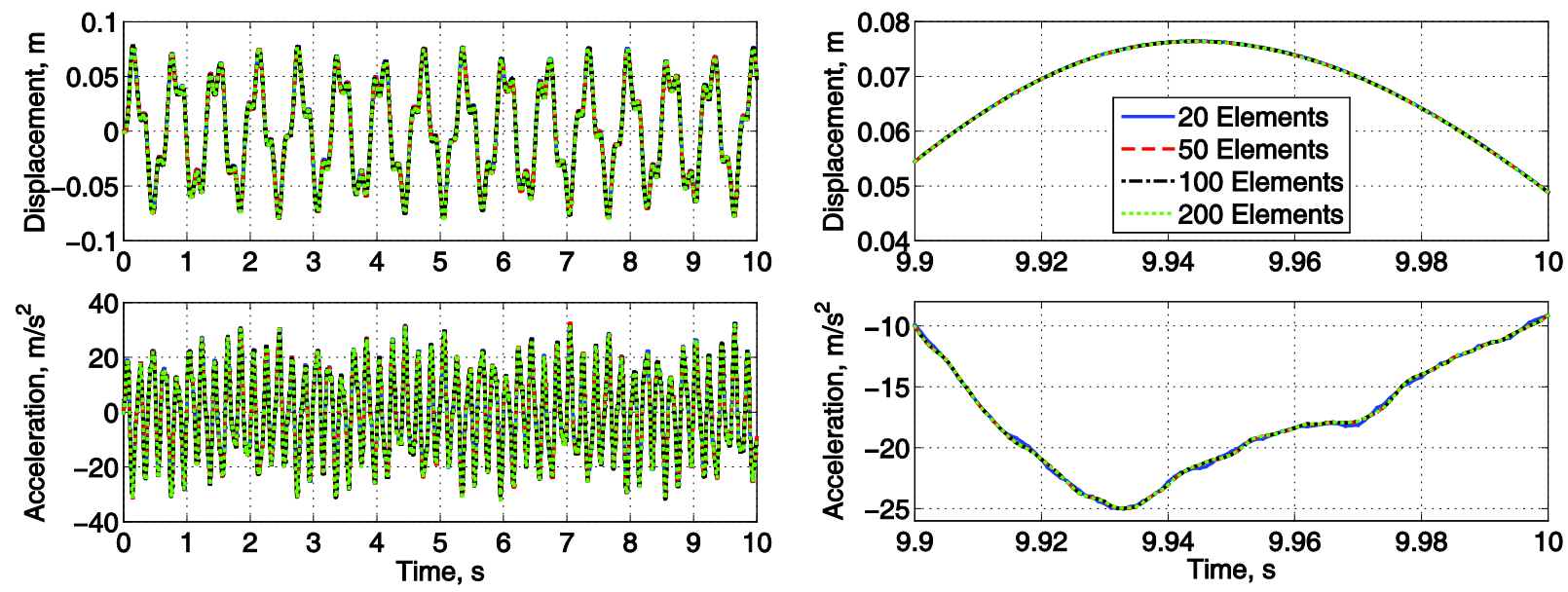

(a) Time: $0-10 \mathrm{~s}$

(b) Time: $9.9-10 \mathrm{~s}$

Figure 11 Beam tip displacement and translational aceeleration from direct integrations of finite element models. 

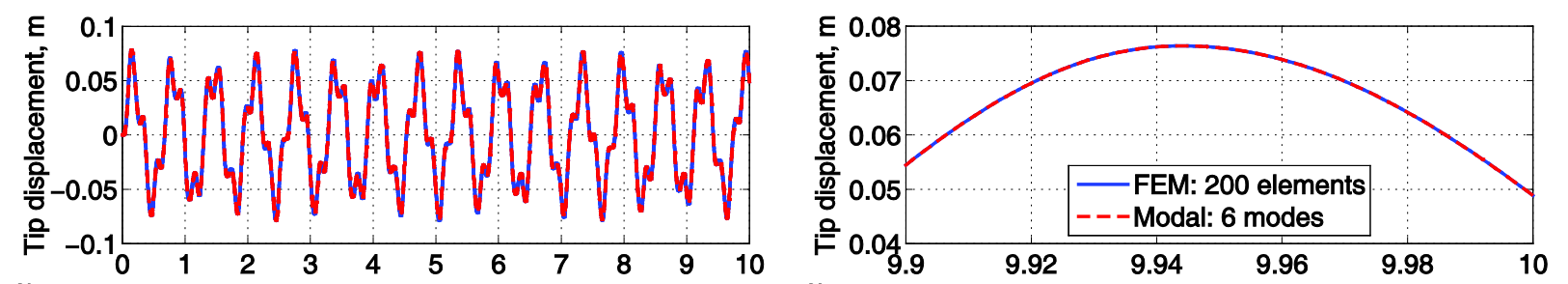

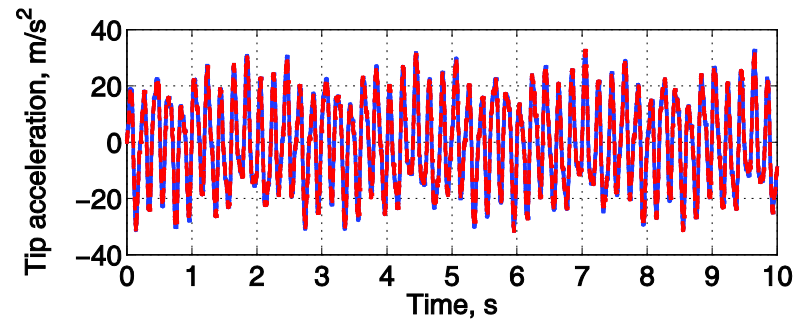

(a) Time: $0-10 \mathrm{~s}$

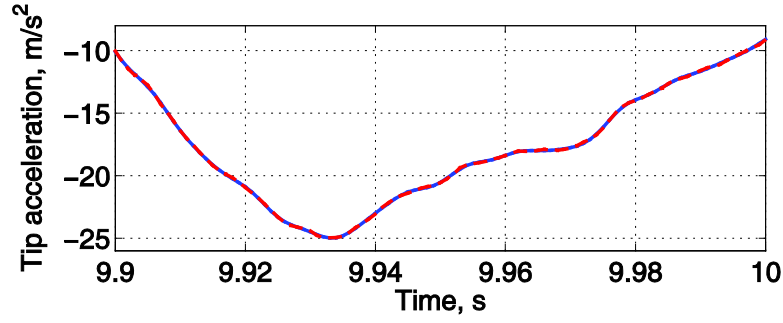

(b) Time: $9.9-10 \mathrm{~s}$

Figure 12 Beam tip displacement and translational aceeleration from finite element and modal solutions.

To solve for the issue, a modal based transient solution can be used. Here, the first 6 modes of the 200 -element finite element model are represented by 20 shifted Legendre polynomials. The resulting modal-based equation is still integrated using the Chen-Ricles scheme. The same time step is used. The CPU time of the simulation is then listed in Table 3. The beam tip displacement and translational acceleration are compared with the ones from the 200element finite element solution (see Fig. 12). From the results, it can be seen that the solution accuracy is well kept in the modal-based solution using the Chen-Ricles scheme and the corresponding CPU time is reduced to allow for real-time studies.

Table 3. CPU time vs. complexity of beam models

\begin{tabular}{cccccc}
\hline \hline Model & 20 elements & 50 elements & 100 elements & 200 elements & $\begin{array}{c}6 \text { modes from } \\
\text { 200 elements }\end{array}$ \\
\hline $\begin{array}{c}\text { Dimension of problem } \\
\text { CPU time }\end{array}$ & 40 & 100 & 200 & 400 & 20 \\
\hline \hline
\end{tabular}

\section{Indirect Solution}

The indirect solution of the beam dynamics is studied in this section. For the indirect solution, the excitation to the beam is unknown. However, intermittent strains along the beam span and a single point angular velocity and translational acceleration are measured by devices of FOS and IMU. The target is to estimate the angular velocity and translational acceleration along the whole beam span.

In the current study, the transient response from the direct solution is used as the "measurement" data, even though the input of the indirect solution should be real measurement data from the sensors. Specifically, the IMU measurements are assumed to be the angular velocity and translational acceleration data taken at the $80 \%$ span from the beam root. Strains are also extracted from the time simulation data at evenly distributed stations along the beam, coincident with the nodes of the finite element models. The spanwise angular velocity and translational acceleration are also going to be recovered at these points.

A first calculation is based on the transient results of the 20-element finite element model. The first 20 shifted Legendre polynomials are used to approximate the first 6 modes. Then the aforementioned approach is used to recover the angular velocity and translational acceleration along the beam. Figure 13 compares the "measured" and fitted strain, as well as the "real" angular velocity and translational acceleration that are actually extracted from the transient simulation and the recovered data, all at $t=4 \mathrm{~s}$. Then the same calculations are repeated using the 50-, 100-, and 200-element models, respectively. The results are all plotted in Figs. 14 to 16. From the results shown in Figs. 13 to 16 , one can see that the simulation data from the 20-element finite element model is sufficient to fit the strain and estimate the angular velocity. However, the estimated translational acceleration is still very off. One has to use the transient data from the refined finite element model, in order to accurately estimate the translational acceleration, as shown in Fig. 16. 

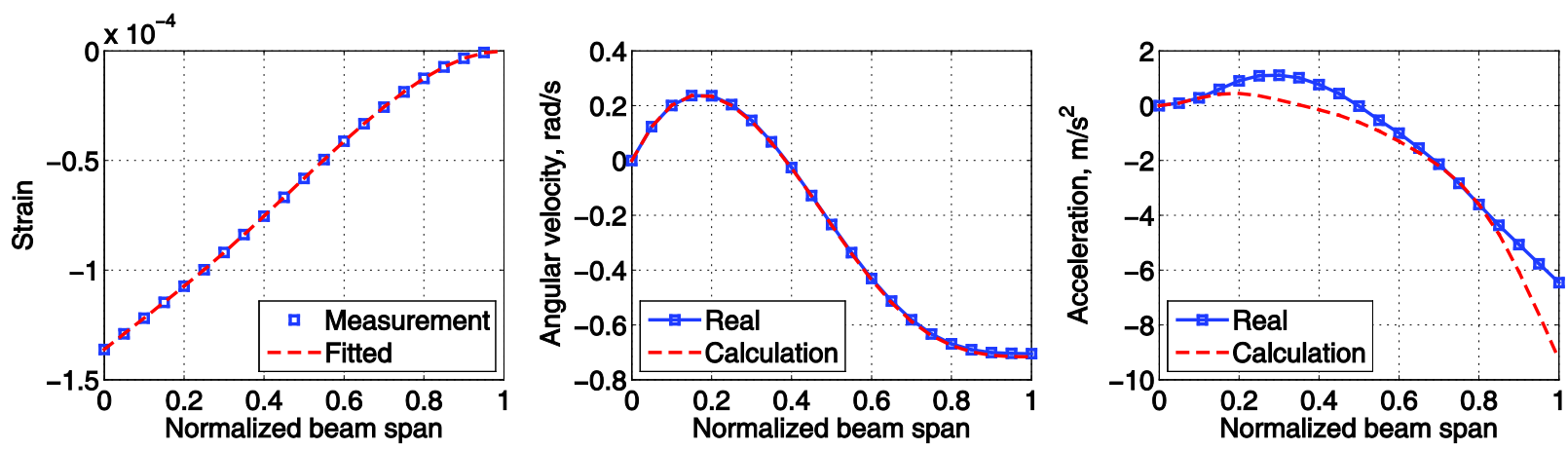

Figure 13 Fitted strain, estimated velocity and acceleration, using 20-element FEM simulation data.
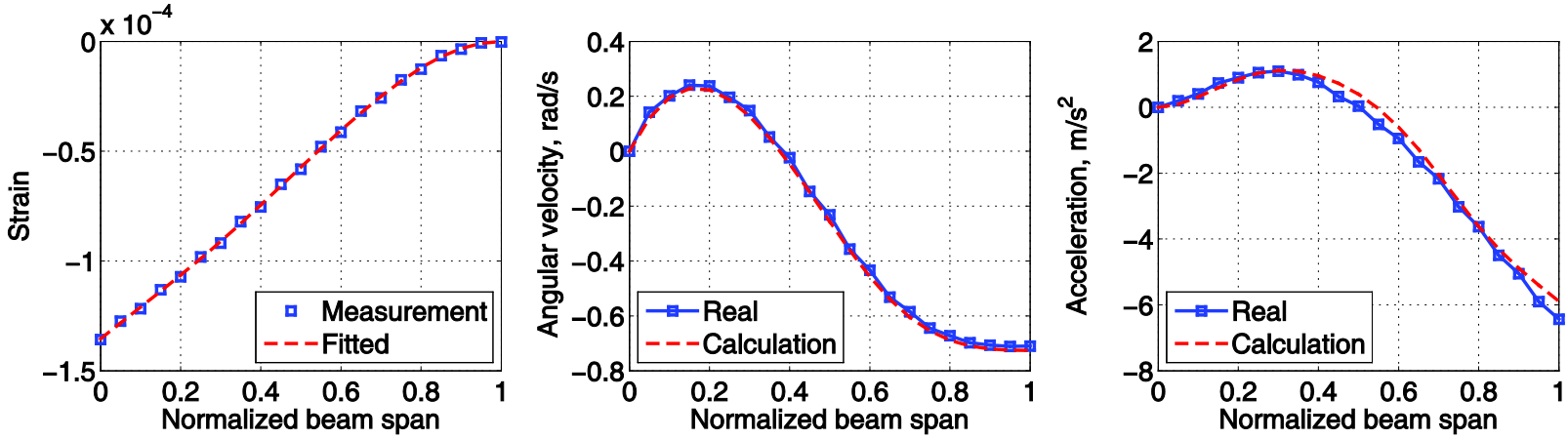

Figure 14 Fitted strain, estimated velocity and acceleration, using 50-element FEM simulation data.
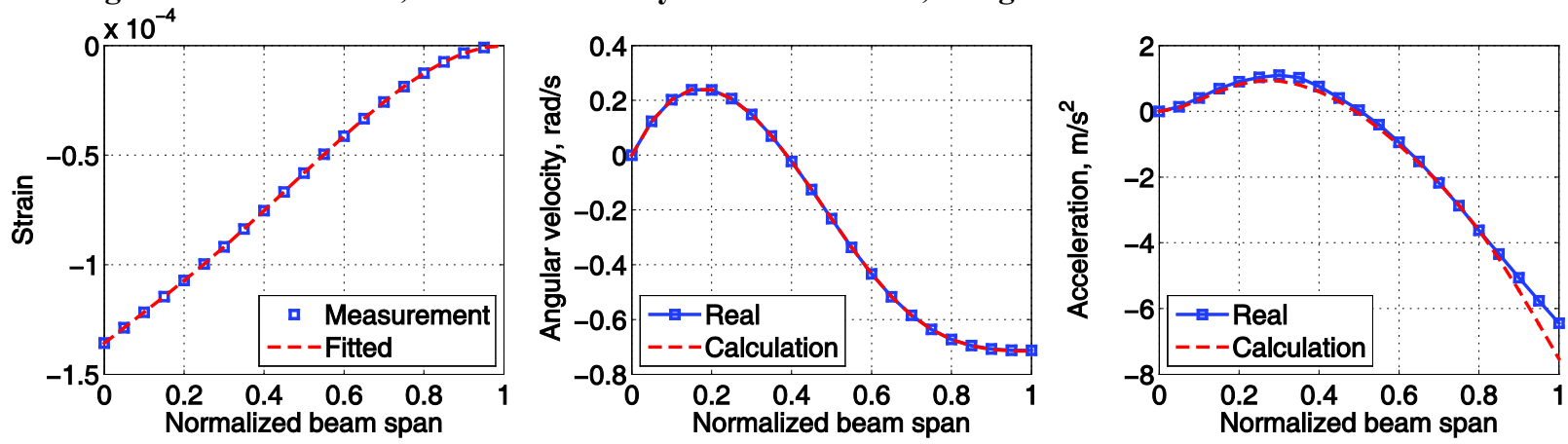

Figure 15 Fitted strain, estimated velocity and acceleration, using 100-element FEM simulation data.
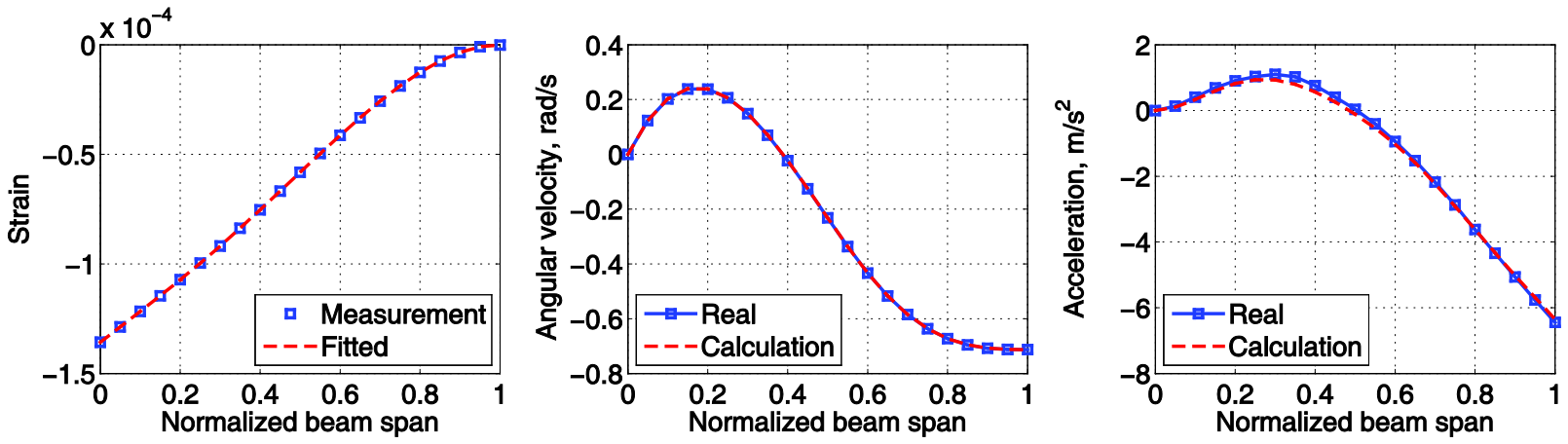

Figure 16 Fitted strain, estimated velocity and acceleration, using 200-element FEM simulation data.

A further study is to learn the impact of the number of modes on the solution's accuracy. In doing so, the transient response from the 200-elelment finite element model is used as the "measurement". However, a different number of modes are used to represent the beam deformation. Here, five different cases involving 2, 4, 6, 9, and 12 
modes, respectively, are studied. All these modes are then approximated by 20 shifted Legendre polynomials. Figures 17 to 19 compare the fitted strains and estimated angular velocities and translational accelerations. It is not a surprise that the 2-mode representation of the beam deformation is almost sufficient to fit the strain (Fig. 17). However, the accurate estimation of the velocity, as well as the acceleration, along the beam needs more modes (Figs. 18 and 19). However, the inclusion of excessive modes may also compromise the solution of acceleration, which is highlighted by the case with 12 modes. Table $\mathbf{4}$ also shows such a trend.

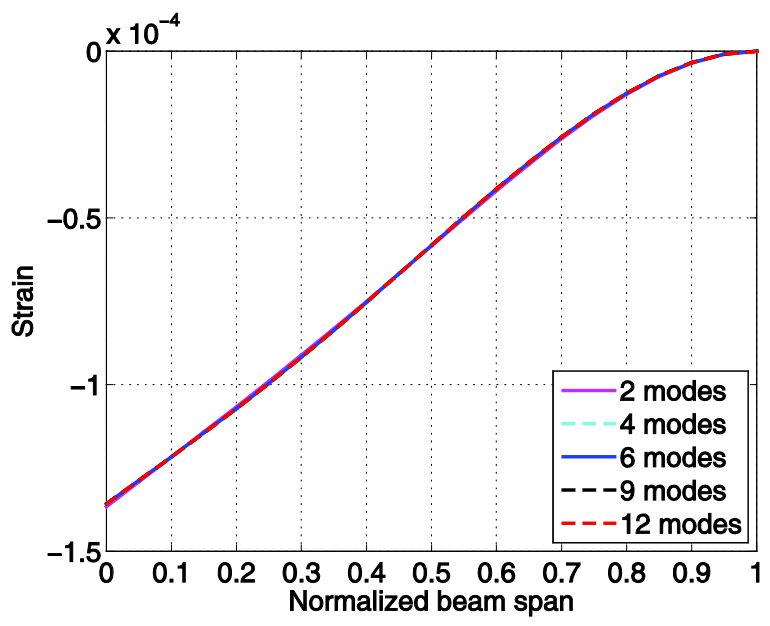

Figure 17 Fitted strain along the beam using diffeent numbers of modes.

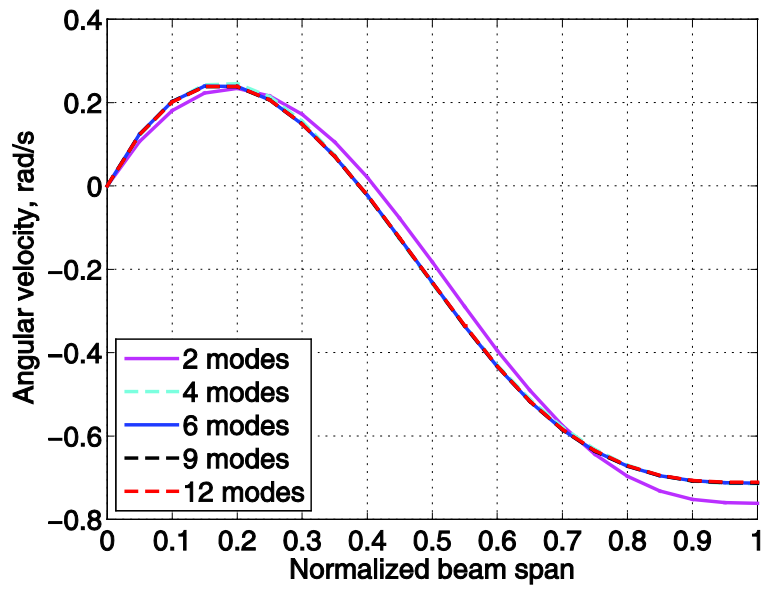

Figure 18 Estimated angular velocity along the beam using diffeent numbers of modes. 


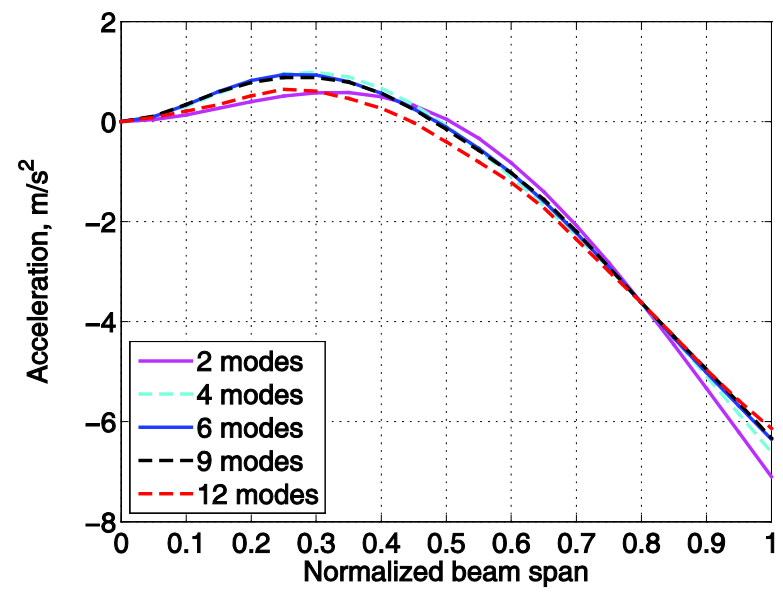

Figure 19 Estimated translational acceleration along the beam using diffeent numbers of modes.

Table 4. RMS error between recovered and real translational accelerations

\begin{tabular}{|c|c|c|c|c|c|c|c|c|c|c|c|c|}
\hline & 1 & 2 & 3 & $\overline{4}$ & $\overline{5}$ & $\overline{6}$ & $\overline{7}$ & 8 & $\overline{9}$ & 10 & 11 & 12 \\
\hline $\mathrm{RM}$ & 1.237 & 0.316 & 0.102 & 0.104 & 0.119 & 0.108 & 0.120 & 0.156 & 0.128 & 0.697 & 0.231 & 0.317 \\
\hline
\end{tabular}

For the indirect solution performed so far, it is all at $t=4 \mathrm{~s}$. The solution process is then repeated in the time range of 0 to 10 seconds. Figures 20 to 22 compare the spanwise bending strain, angular velocity, and translational acceleration between the indirect solution and the "measurement" or "actual" data (essentially results from the direct time integration). Figure 23 provides the RMS error of the solution of translational acceleration at the locations of all FOSs. From the results, it is evident that the accuracy of the indirect solution is well maintained throughout the time range.

Finally, a damped system is considered for the beam, where the stiffness-proportional damping coefficient $\alpha$ is assumed to be 0.002 . Then the bending strain along the beam (as the FOS measurements) as well as the angular velocity and translational acceleration at the $80 \%$ beam span (as the IMU measurements) are extracted and serve as the input to the indirect solution. The indirect solution process is also repeated in the time range of 0 to 10 seconds. Figures 24 to 26 compare the spanwise bending strain, angular velocity, and translational acceleration between the indirect solution and the "measurement" or "actual" data (essentially results from the direct time integration). Figure 27 provides the RMS error of the solution of translational acceleration at the locations of FOSs. From the side to side comparisons, one can see the indirect solution approach is also applicable to damped systems, even though the damping was not considered in the original development of the indirect solution process.

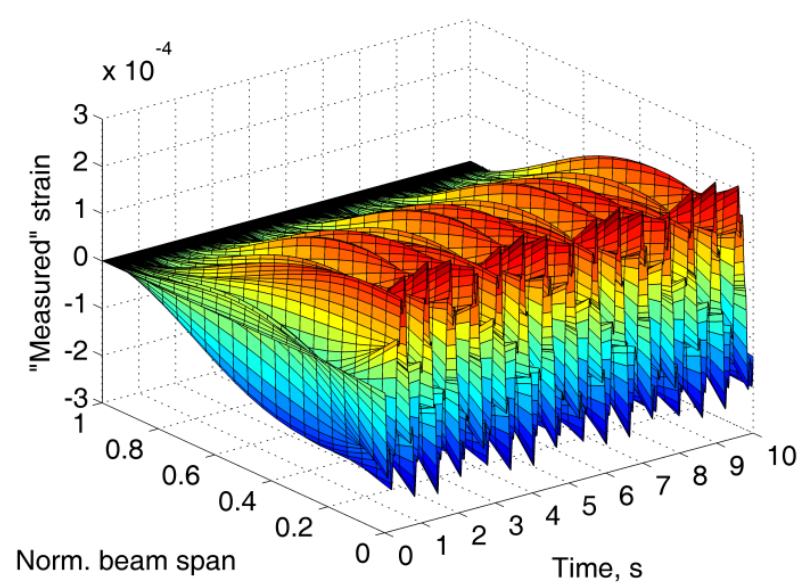

(a) "Measured" strain

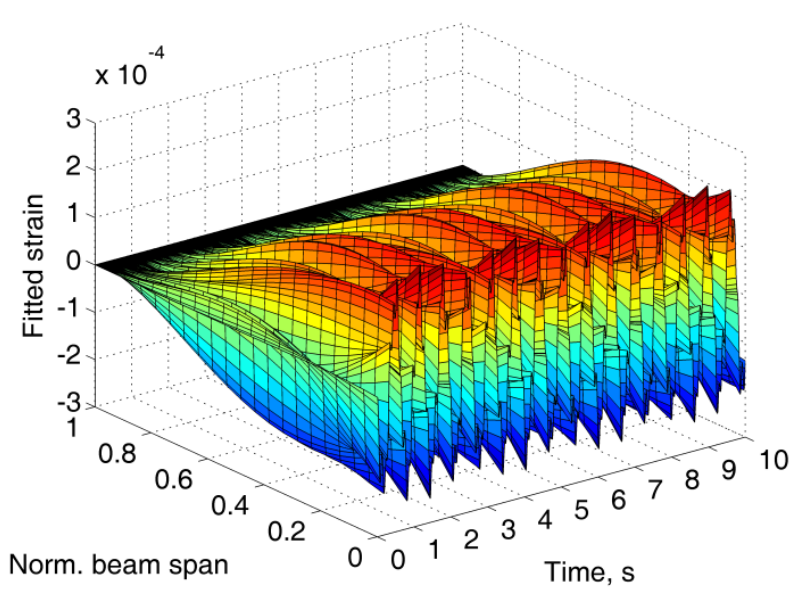

(b) Fitted strain

Figure 20 "Measured" and fitted bending strain along the beam from 0 to $10 \mathrm{~s}$. 


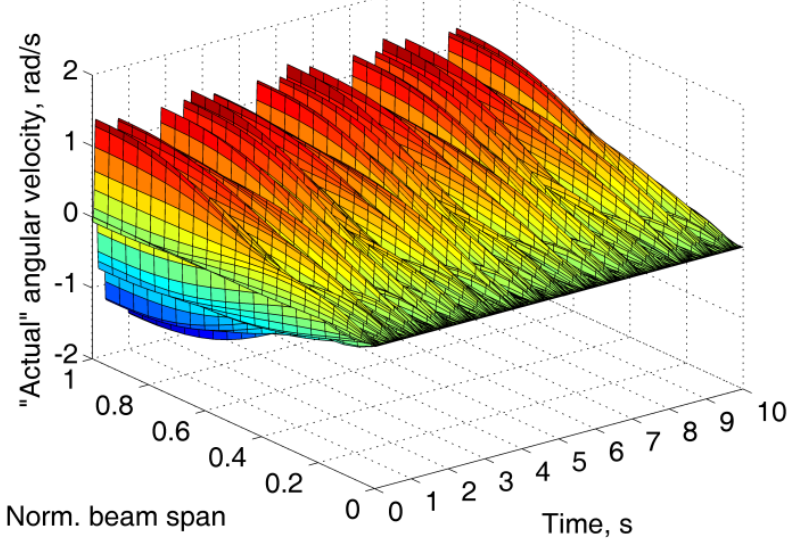

(a) "Actual" angular velocity

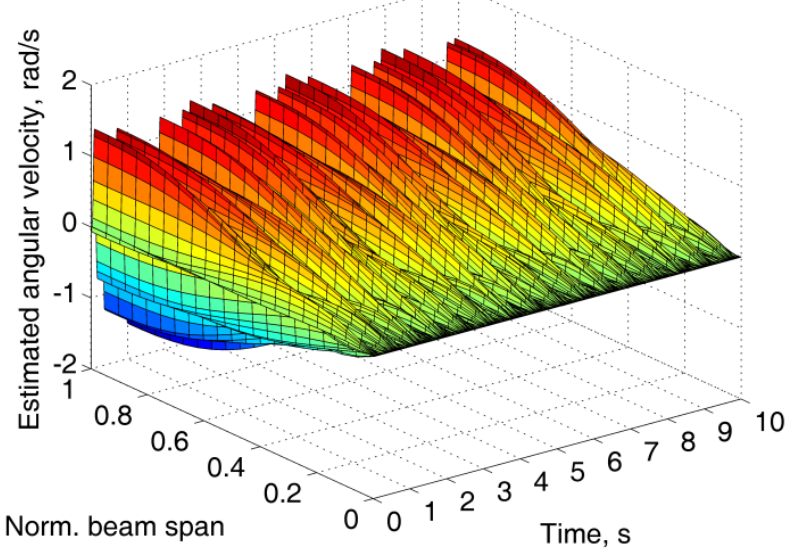

(b) Estimated angular velocity

Figure 21 "Actual" and estimated angular velcoity along the beam from 0 to $10 \mathrm{~s}$.

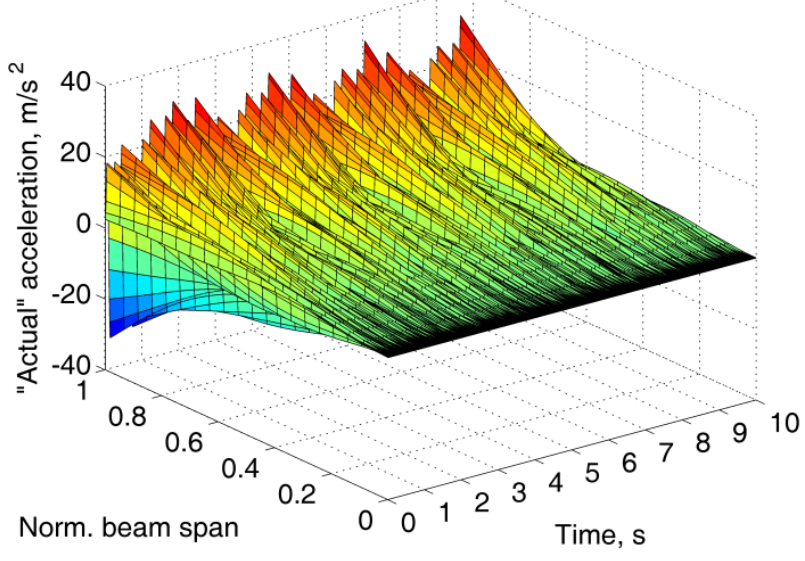

(a) "Actual" translational acceleration

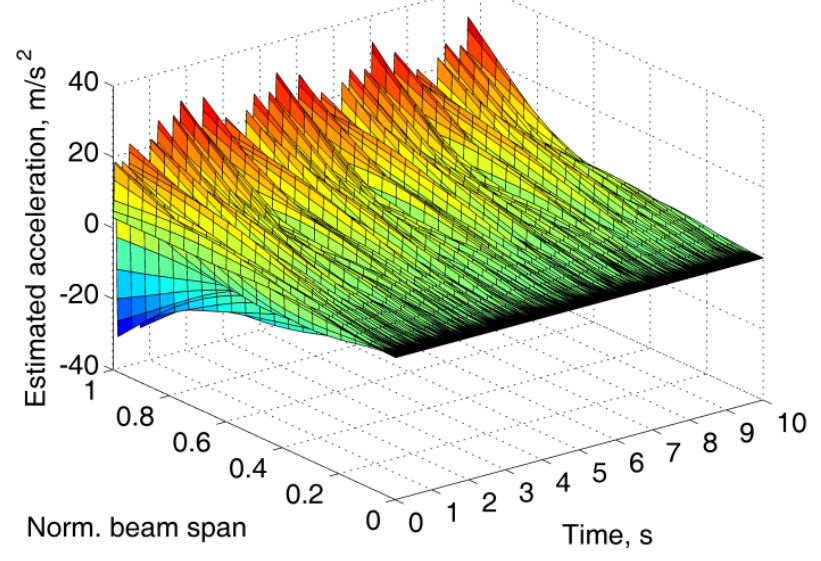

(b) Estimated translational acceleration

Figure 22 "Actual" and estimated translational acceleration along the beam from 0 to $10 \mathrm{~s}$.

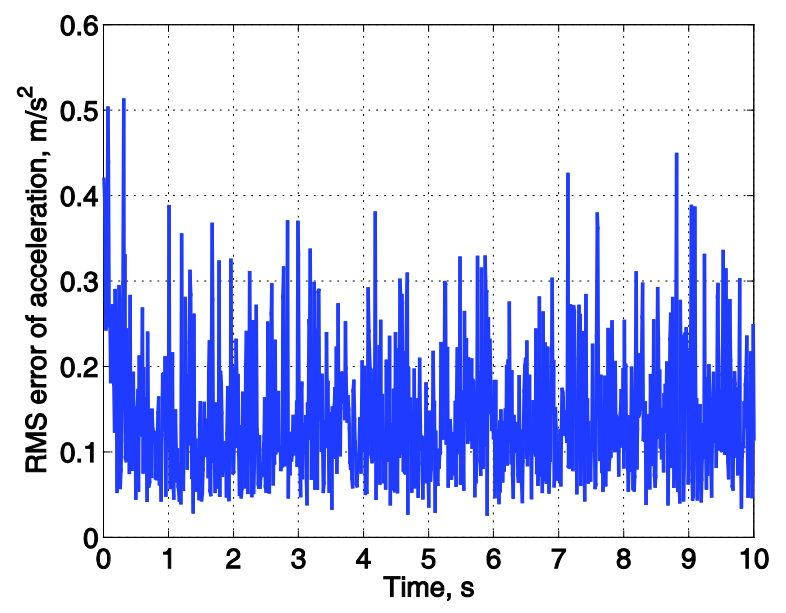

Figure 23 RMS error of tranlational acceleration between the estimation and actual soltiuon without structural damping. 


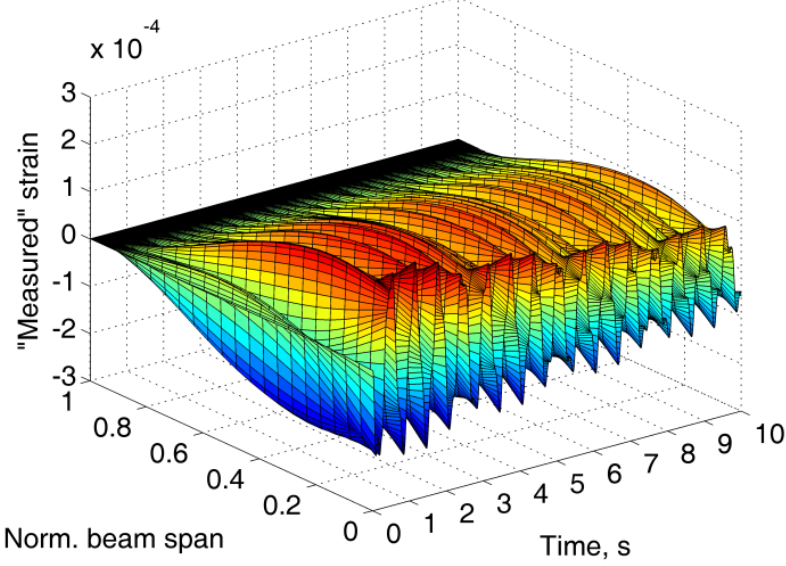

(a) "Measured" strain

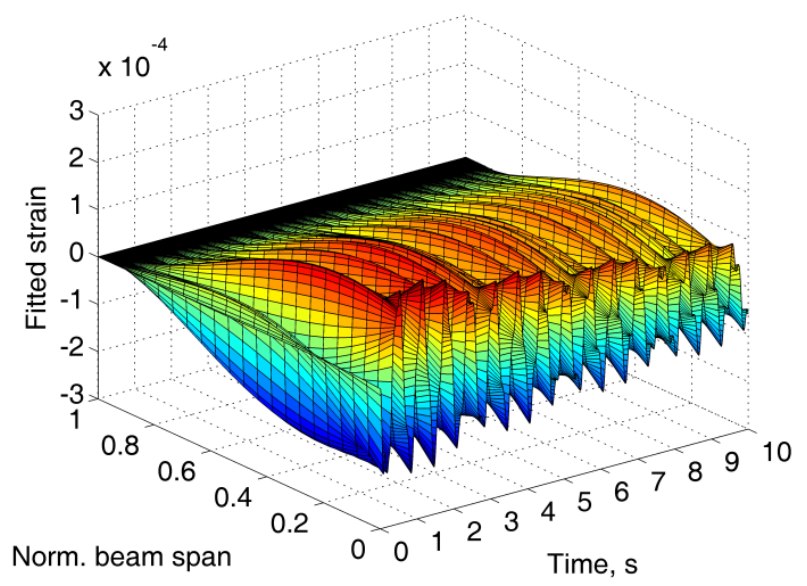

(b) Fitted strain

Figure 24 "Measured" and fitted strain along the beam from 0 to $10 \mathrm{~s}$, with structural damping.

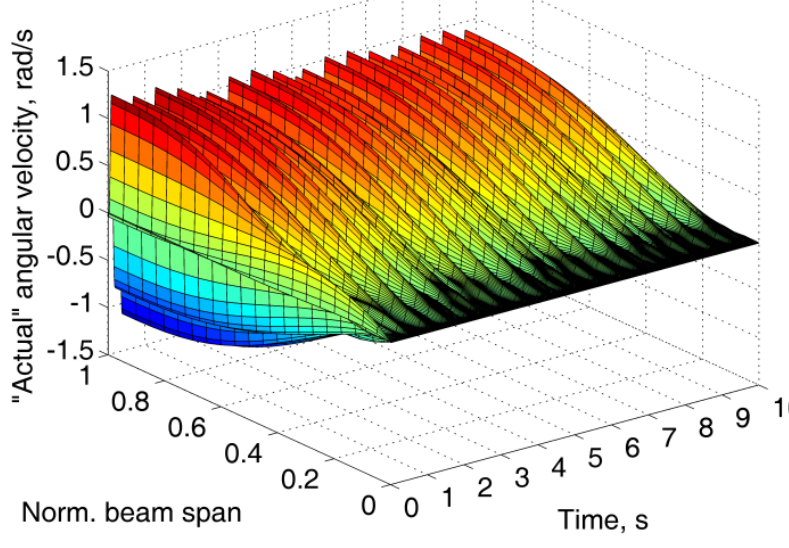

(a) "Actual" angular velocity

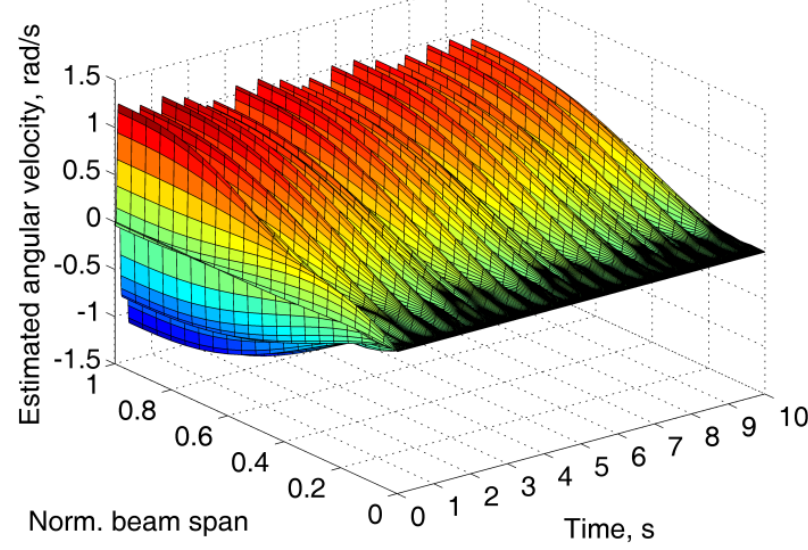

(b) Estimated angular velocity

Figure 25 "Actual" and estimated angular velocity along the beam from 0 to $10 \mathrm{~s}$, with structural damping.

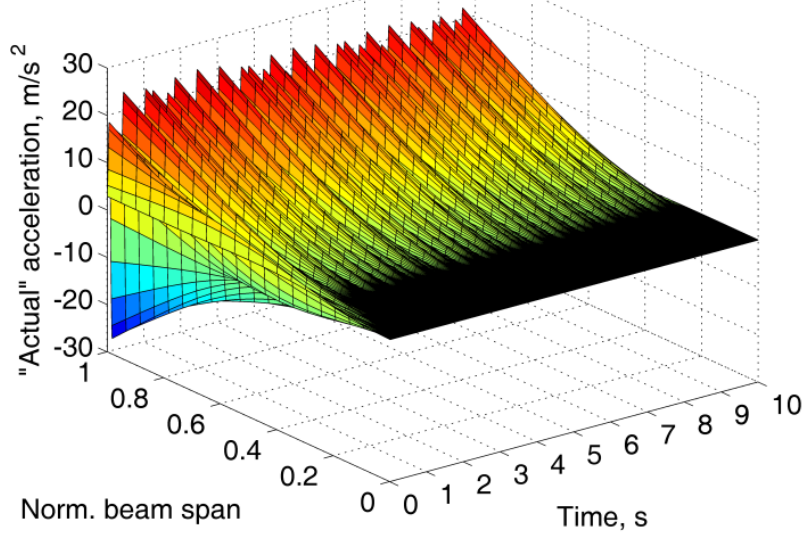

(a) "Actual" translational acceleration

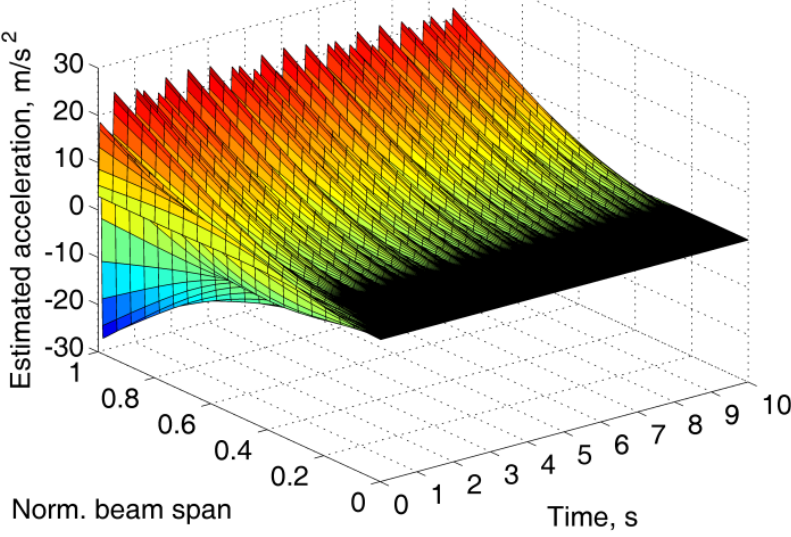

(b) Estimated translational acceleration 
Figure 26 "Actual" and estimated translational acceleration along the beam from 0 to $10 \mathrm{~s}$, with structural damping.

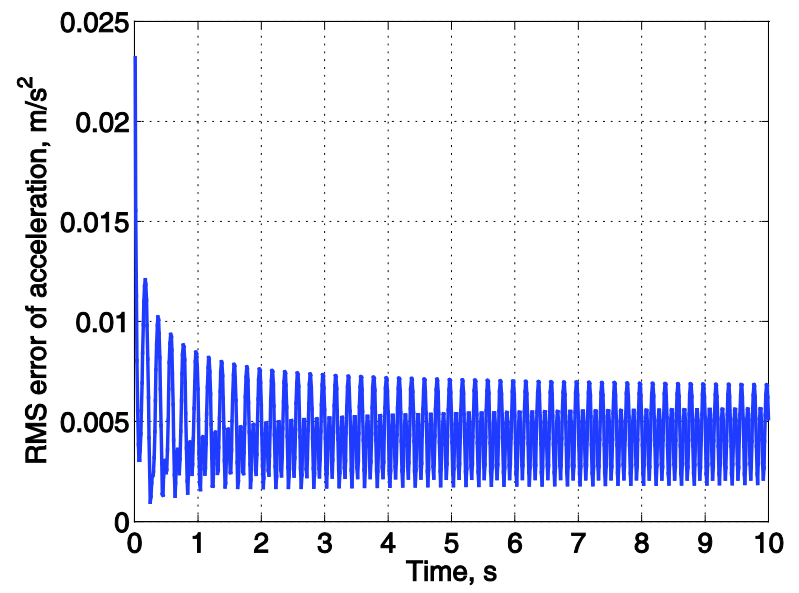

Figure 27 RMS error of tranlational acceleration between the estimation and actual soltiuon with structural damping.

\section{Conclusion}

Based on the Euler-Bernoulli beam theory, analytical and numerical approaches were derived and implemented in this paper for real-time solutions of the bending dynamics of flexible rockets. The finite element discretization of the beam model was created at the beginning, where the discrete mode shapes were extracted and represented by using the continuous shifted Legendre polynomials. This treatment allows for the spatial derivatives of the mode shapes in order to represent the rotation and curvature modes, which are generally difficult to work directly on the discrete mode shapes of the finite element model.

By implementing an explicit Newmark-based scheme, the direct time integration of the beam bending equation can be finished in the real time. This helps the future real-time simulations and control development of flexible launch vehicles.

In real-time control operations of flexible rockets, the external excitation to the vehicle may not be known. To enable to control of the flexible rockets in real time, an indirect solution of beam bending dynamics was also explored in the paper, where only limited beam bending strains and kinematic quantities were measured. To find the distributed bending dynamics along the beam, the modal magnitude of the beam was solved, by taking advantage of the approximation of the modes using the Legendre polynomials, subject to the available sensor measurements. A backward finite-difference was used to represent the rate and acceleration of the modal magnitudes. This study successfully established a quick, non-iterative, "analytical" solution of the beam dynamics, based on the available sensor measurements. Each of the solution can be done in about $10^{-3} \mathrm{~s}$, which satisfied the requirement of further real-time control developments. The solution was accurate and stable for perfect measurement data, since the spanwise angular velocity and translational acceleration were both precisely estimated. However, further studies are necessary to consider the solution based on the noised measurement data. In summary, the derived formulations are capable of handling: (1) non-uniform beam stiffness, (2) non-uniform inertia distribution, (3) different IMU locations, (4) various boundary conditions, and (5) potential 2- or 3-DOF beam bending and torsion.

As some future developments, one can consider the coupled beam in-plane/out-of-plane bending and torsion solutions to provide a more accurate modeling of launch vehicle dynamics, which involves more complex modes. At the same time, the coupling of the elastic beam modes with the 6-DOF rigid-body rotations needs to be considered. This requires more complex boundary conditions for the beam.

\section{Acknowledgments}

This work is supported by the NASA Launch Services Program Special Study LSP-14-015. Cecilia King acknowledges the support of the Graduate Council Fellowship from the University of Alabama. Technical discussions on the numerical integration scheme with Dr. Wei Song (Civil Engineering, University of Alabama) are 
gratefully acknowledged. The views expressed in this article are those of the authors and do not reflect the official policy or position of NASA or the U.S. Government.

\section{References} 147.

${ }^{1}$ Leitmann, G., "On the Equation of Rocket Motion," Journal of the British Interplanetary Society, Vol. 16, 1957, pp. 141-

${ }^{2}$ Stengel, R. F., "Flight Performance of a Small, Low-Altitude Rocket," Journal of Spacecraft and Rockets, Vol. 3, No. 6, 1966, pp. 938-939.

${ }^{3}$ Vinh, N. X., "General Theory of Optimal Trajectory for Rocket Flight in a Resisting Medium," Journal of Optimization Theory and Applications, Vol. 11, No. 2, 1973, pp. 189-202.

${ }^{4}$ Eke, F. O. and Cervantes, E., "Dynamics of Axisymmetric Rockets in Free Flight," Journal of Dynamic Systems Measurement and Control-Transactions of the ASME, Vol. 120, No. 3, 1998, pp. 410-414.

${ }^{5}$ Lee, B. S., Choi, J. H. and Kwon, O. J., "Numerical Simulation of Free-Flight Rockets Air-Launched from a Helicopter," Journal of Aircraft, Vol. 48, No. 5, 2011, pp. 1766-1775.

${ }^{6}$ Anderson, J. D., Introduction to Flight (3rd Edition), McGraw-Hill Series in Aeronautical and Aerospace Engineering, McGraw-Hill, Inc., New York, NY, 1989.

${ }^{7}$ Reis, G. E. and Sundberg, W. D., "Calculated Aeroelastic Bending of a Sounding Rocket Based on Flight Data," Journal of Spacecraft and Rockets, Vol. 4, No. 11, 1967, pp. 1489-1494.

${ }^{8}$ Huang, X. and Zeiler, T. A., "Dynamics of Flexible Launch Vehicles with Variable Mass," AIAA-2006-0826, 44th AIAA Aerospace Sciences Meeting and Exhibit, Reno, NV, Jan. 9-12, 2006.

${ }^{9} \mathrm{Hu}$, P. and Ren, G., "Multibody Dynamics of Flexible Liquid Rockets with Depleting Propellant," Journal of Guidance Control and Dynamics, Vol. 36, No. 6, 2013, pp. 1849-1858.

${ }^{10}$ Choi, H. D. and Bang, H., "An Adaptive Control Approach to the Attitude Control of a Flexible Rocket," Control Engineering Practice, Vol. 8, No. 9, 2000, pp. 1003-1010.

${ }^{11}$ Shearer, C. M. and Cesnik, C. E. S., "Modified Generalized- $\alpha$ Method for Integrating Governing Equations of Very Flexible Aircraft," AIAA-2006-1747, 47th AIAA / ASME / ASCE / AHS / ASC Structures, Structural Dynamics, and Materials Conference, Newport, RI, May 1-4, 2006.

${ }^{12}$ Chen, C. and Ricles, J. M., "Development of Direct Integration Algorithms for Structural Dynamics Using Discrete Control Theory," Journal of Engineering Mechanics-ASCE, Vol. 134, No. 8, 2008, pp. 676-683.

${ }^{13}$ Li, J., Kapania, R. K. and Spillman, W. B., Jr., "Placement Optimization of Distributed-Sensing Fiber-Optic Sensors using Genetic Algorithms," Aiaa Journal, Vol. 46, No. 4, 2008, pp. 824-836.

14"Dryden Fiber Optic Sensing Technology Suite."

${ }^{15}$ Wolf, M. G., Griffin, E. D., Gutierrez, H., Suhey, J. D., Su, W. and Stanley, J. E., "Flexible Body Control using Fiber Optic Sensors (FlexFOS)," NASA Report 1426097577, NASA Technology Transfer System, 2015.

${ }^{16}$ Abramowitz, M. and Stegun, I. A., Handbook of Mathematical Functions, Dover Publications, New York, NY, 1968.

${ }^{17}$ Chen, C. and Ricles, J. M., "Stability Analysis of Direct Integration Algorithms Applied to Nonlinear Structural Dynamics," Journal of Engineering Mechanics-ASCE, Vol. 134, No. 9, 2008, pp. 703-711. 